University of Redlands

\title{
A Prototype Path Prediction Tool
}

\author{
A Major Individual Project submitted in partial satisfaction of the requirements \\ for the degree of Master of Science in Geographic Information Systems \\ by \\ Andrew Woizesko \\ Ruijin MA, Ph.D., Committee Chair \\ Douglas Flewelling, Ph.D.
}

December 2012 
A Prototype Path Prediction Tool

Copyright (C) 2012

by

Andrew Woizesko 
The report of Andrew Woizesko is approved.
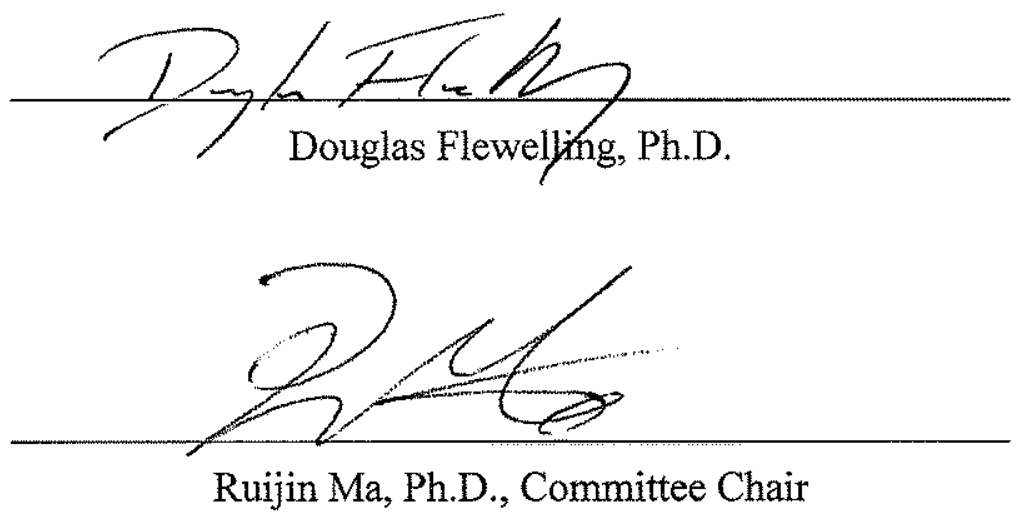

December 2012 



\section{Acknowledgements}

I would like to thank my parents for supporting me through this project, the master's program, and throughout my life. Without them I would not be where I am today. These few sentences do not express my appreciation for them. I would also like to thank Ruijin Ma; he was an immensely helpful when discussing and working-out problems with the project. I would like to thank Doug Flewelling, Mark Kumler, and Fang Ren for their contributions to both the project and my education. Finally, I want to thank Debbie Riley for everything she does with the MS GIS program, she kept me on track, and generally made everything more pleasant. 



\author{
Abstract \\ A Prototype Path Prediction Tool \\ By \\ Andrew Woizesko
}

\begin{abstract}
When a person moves through an environment they will decide where to travel based on their interpretation of the surroundings. This becomes important in search and rescue scenarios and military operations when a person's route is of interest. One solution to predict this route is to model the way people travel. This paper documents the process of developing a prototype path prediction tool using the Python scripting language and ArcMap tools. The general model approach was to create a simulation based on a least cost path analysis restricted by viewshed analysis. While the concept is straightforward, creating the program proved complex due to the management of vector and raster data, and accounting for numerous application possibilities and variable combinations. The result was a durable simulation capable of incorporating a directional bias, observer height, travel speed, and the ability to accommodate a level of randomness.
\end{abstract}





\section{Table of Contents}

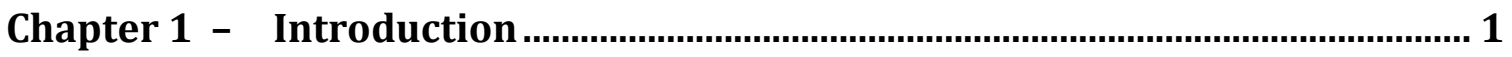

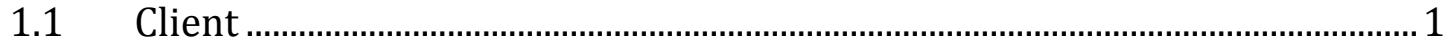

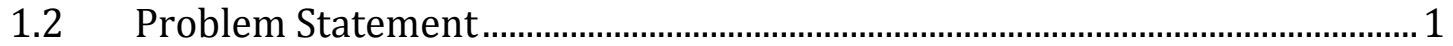

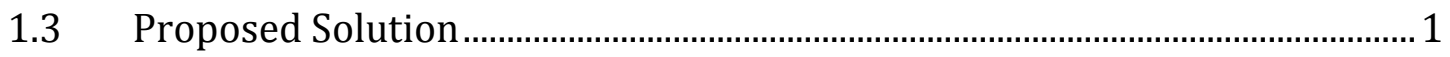

1.3.1 Goals and Objectives ……………………………………………………….... 1

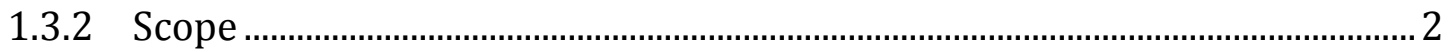

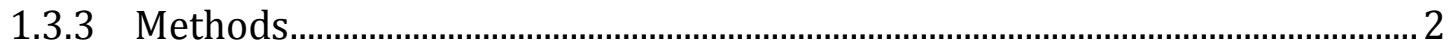

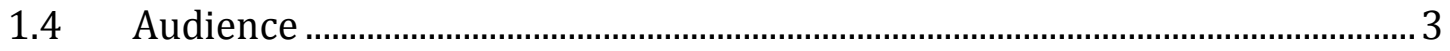

1.5 Overview of the Rest of this Report .................................................................... 3

Chapter 2 - Background and Literature Review................................................. 5

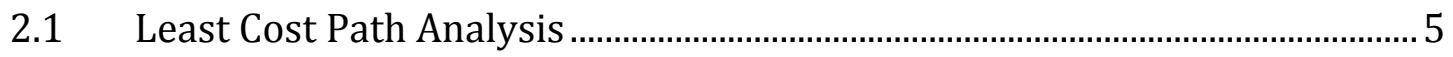

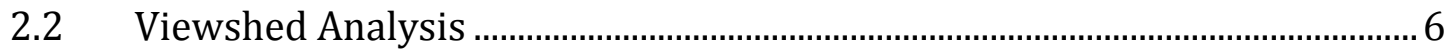

$2.3 \quad$ DEM and TIN Considerations ............................................................................

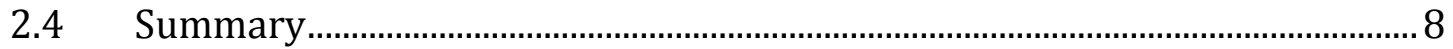

Chapter 3 - Systems Analysis and Design …......................................................... 9

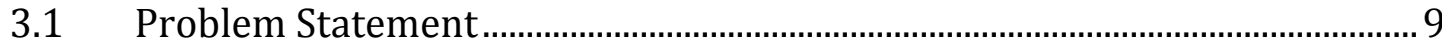

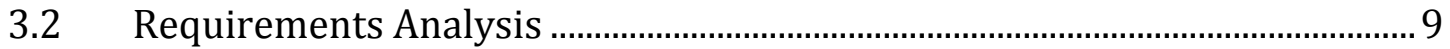

3.3 System Design .................................................................................................. 12

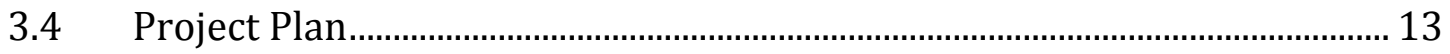

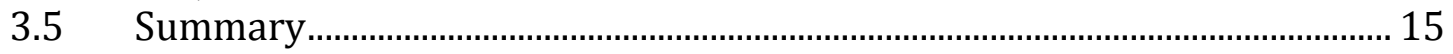

Chapter 4 - Database Design ...............................................................................17

4.1 Conceptual Data Model .............................................................................. 17

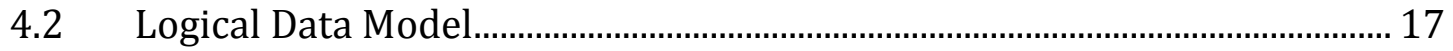

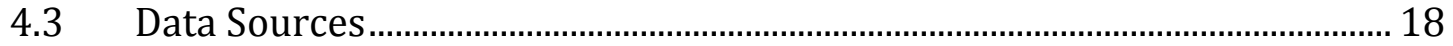

4.4 Data Collection Methods..................................................................................... 18

4.5 Data Scrubbing and Loading ......................................................................... 18

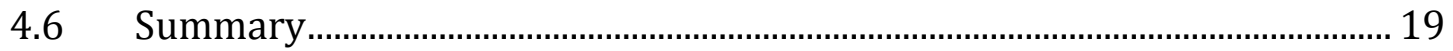

Chapter 5 - Implementation …......................................................................21

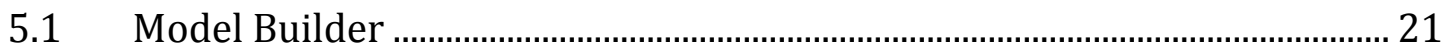

5.2 Modules, Variables, and Environments ......................................................... 22

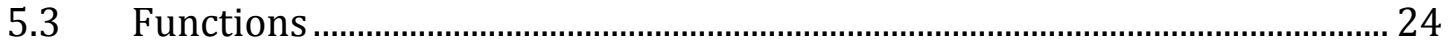

5.4 Initial Input Modifications …………………………………………………. 26

5.5 The Iterative Process ………………………………………………………. 27

5.6 Simulation Completion.................................................................................... 33

Chapter 6 - Results and Analysis...................................................................... 


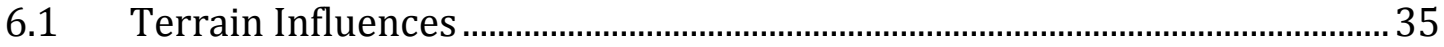

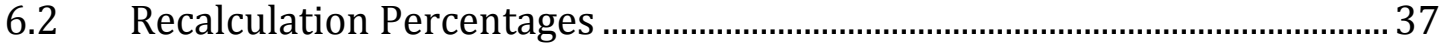

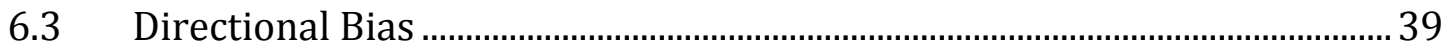

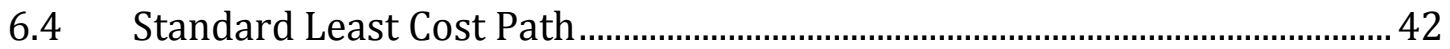

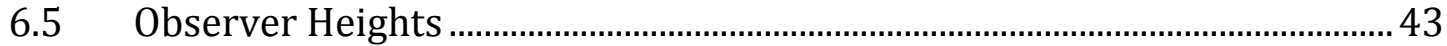

6.6 Problems with Viewshed Analysis .................................................................... 43

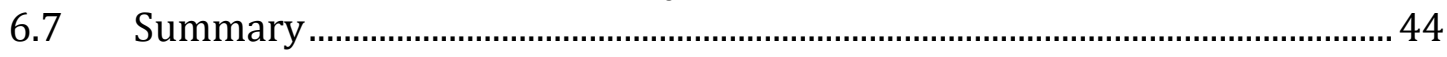

Chapter 7 - Conclusions and Future Work ......................................................45

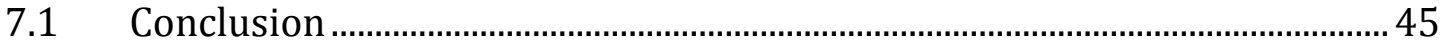

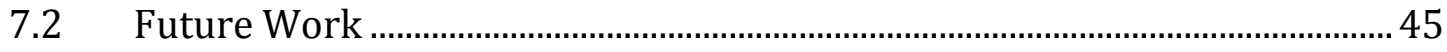

Works Cited ......................................................................................................................47

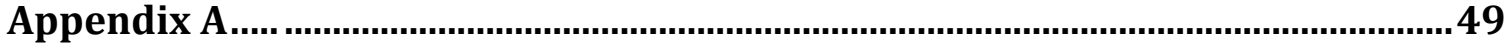




\section{Table of Figures}

Figure 1-1: The study area. ................................................................................. 2

Figure 3-1: The influences of directional bias. .................................................... 11

Figure 3-2: General system design. .................................................................... 13

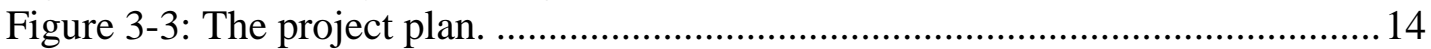

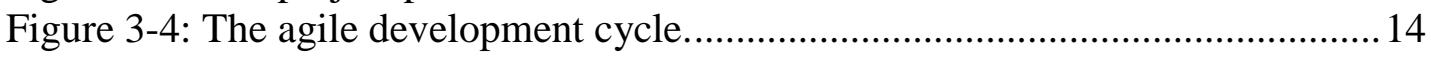

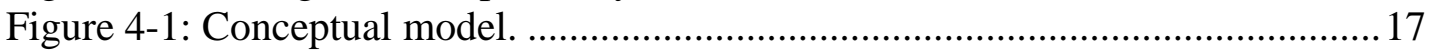

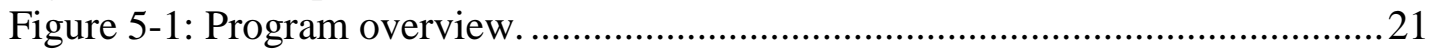

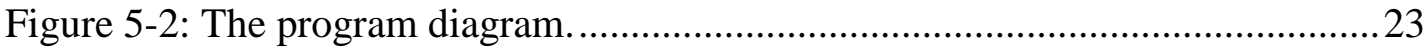

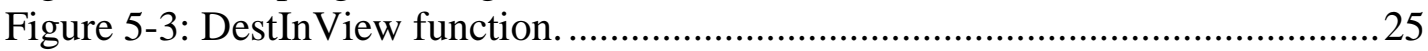

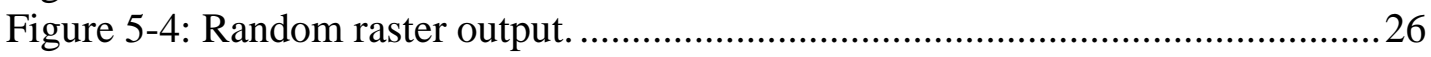

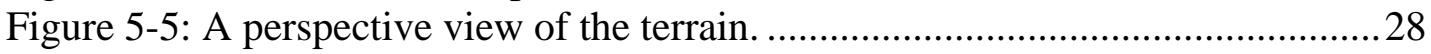

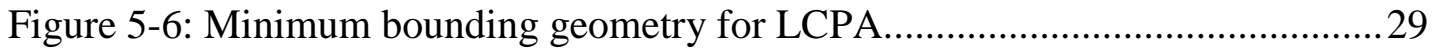

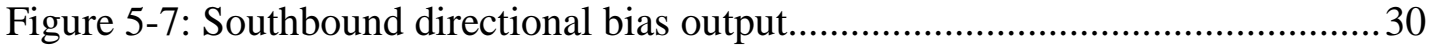

Figure 5-8: A viewshed-restricted cost surface. ................................................... 31

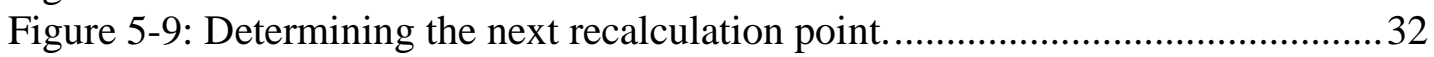

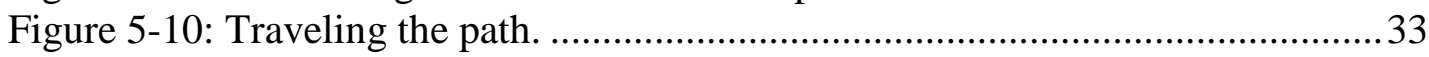

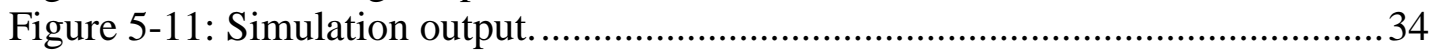

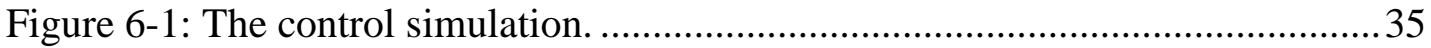

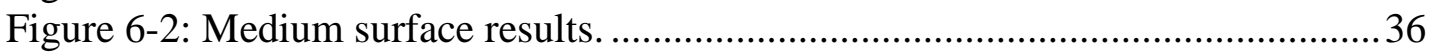

Figure 6-3: Flat surface results. .......................................................................... 37

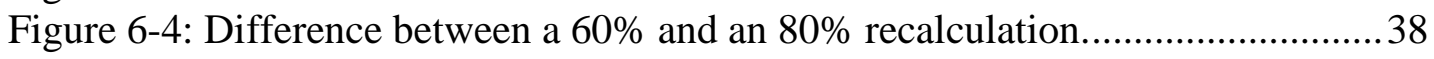

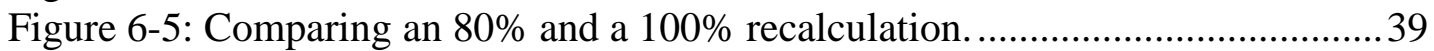

Figure 6-6: Comaparing 60\% recalculation with a directional bias. ....................... 40

Figure 6-7: Comaparing $80 \%$ recalculation with a directional bias. ......................... 41

Figure 6-8: Comaparing 100\% recalculation with a directional bias. ....................... 41

Figure 6-9: Comparing an 80\% recalculation to a standard LCPA. .........................42

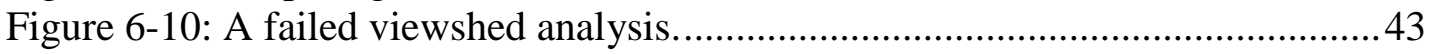

Figure A-1: Code used to verify VSA results......................................................49

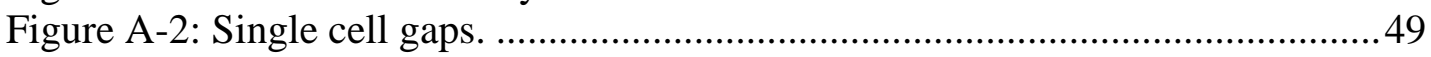

Figure A-3: Code used to patch gaps in the determined path................................50 



\section{List of Tables}

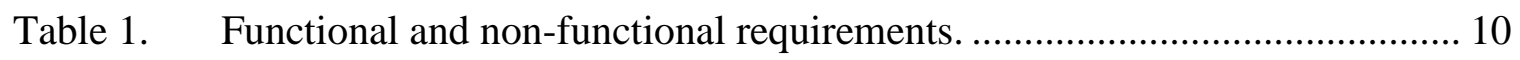

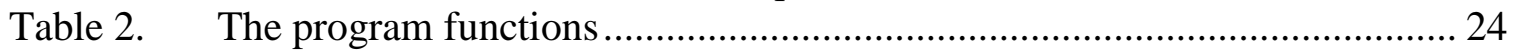

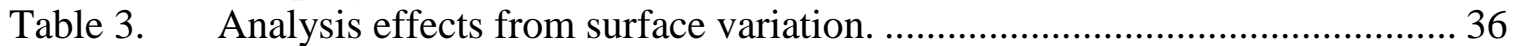

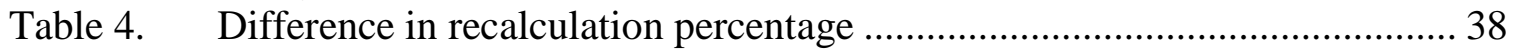

Table 5. Comparing paths with and without directional bias. ................................. 40

Table 6. Comparing a standard LCPA with the control simulation ........................... 42

Table 7. Comparing simulations with different observer heights............................ 43 



\section{List of Acronyms and Definitions}

Cost surface - a grid file used to interpret the environment to the computer in terms of expenditure

DEM - digital elevation model

For loop - a statement in a programming language that allows code to be repeatedly executed for a number of iterations

Function - a named section of a program that performs a specific task

LCPA - least cost path analysis

TIN - triangulated irregular network

VSA - viewshed analysis 



\section{Chapter 1 - Introduction}

There are many instances where it would be beneficial to have an idea of where a person or group will travel. Making such a prediction, however, can be extremely complicated because every person is different and behaviors change over time. This paper details the development of a prototype tool for path prediction.

This chapter introduces the background regarding the construction of a predictive human travel model. Section 1.1 discusses the client and his role in the project; Section 1.2 states the problem being addressed; Section 1.3 provides an overview of the proposed solution; Section 1.4 describes the intended audience; and Section 1.5 describes the structure of the document.

\subsection{Client}

The client for this project is Christopher Price, a GIS Analyst for the United States Government. Mr. Price analyzes international events and needed a tool to assist him in deciding where to allocate resources for military and rescue operations. Specifically, he needed to predict how people will travel through an unfamiliar environment so necessary measures can be taken.

\subsection{Problem Statement}

Without knowing the trends or paths people might utilize when traversing unfamiliar terrain, government agencies face great challenges in allocating resources efficiently and effectively to reach the targeted people. This type of situation occurs when people are lost during hiking or moving to avoid conflict. Agencies need a tool to predict people's travel path given a set of parameters.

\subsection{Proposed Solution}

The solution to the problem was to develop a path prediction tool by simulating human behavior and decision making when a person travels through an environment. The model was scripted with Python and utilized tools from the ArcMap 10.0 module in an iterative process. The model determined the path of least resistance based on the visible area to a destination to identify travel corridors - areas with a high probability of travel.

\subsubsection{Goals and Objectives}

The goal was to help allocate resources effectively and efficiently by providing an accurate travel prediction tool. To achieve this goal, the first objective was to create a cost surface that accurately conveyed human perception of the environment. The next objective was to create a tool that can be used in ArcMap to predict a path a traveler will follow based on their interpretation of their surroundings. The tool should utilize the cost surface and incorporate visibility, speed, direction, and erratic behavior in the prediction. 


\subsubsection{Scope}

The scope of this project included multiple parts. First a cost surface was created based on slope since people generally prefer to travel over smooth terrain when given the opportunity. Since human decision-making incorporates a number of sensory inputs, only subsets of these factors were included: visibility, speed, direction, and erratic behavior. With these inputs, the simulation required testing to ensure durability and to evaluate the validity of the path prediction. Project testing focused on a region of Southern California (Figure 1-1) that contained a variety of obstacles and surface variations to test the simulation response.

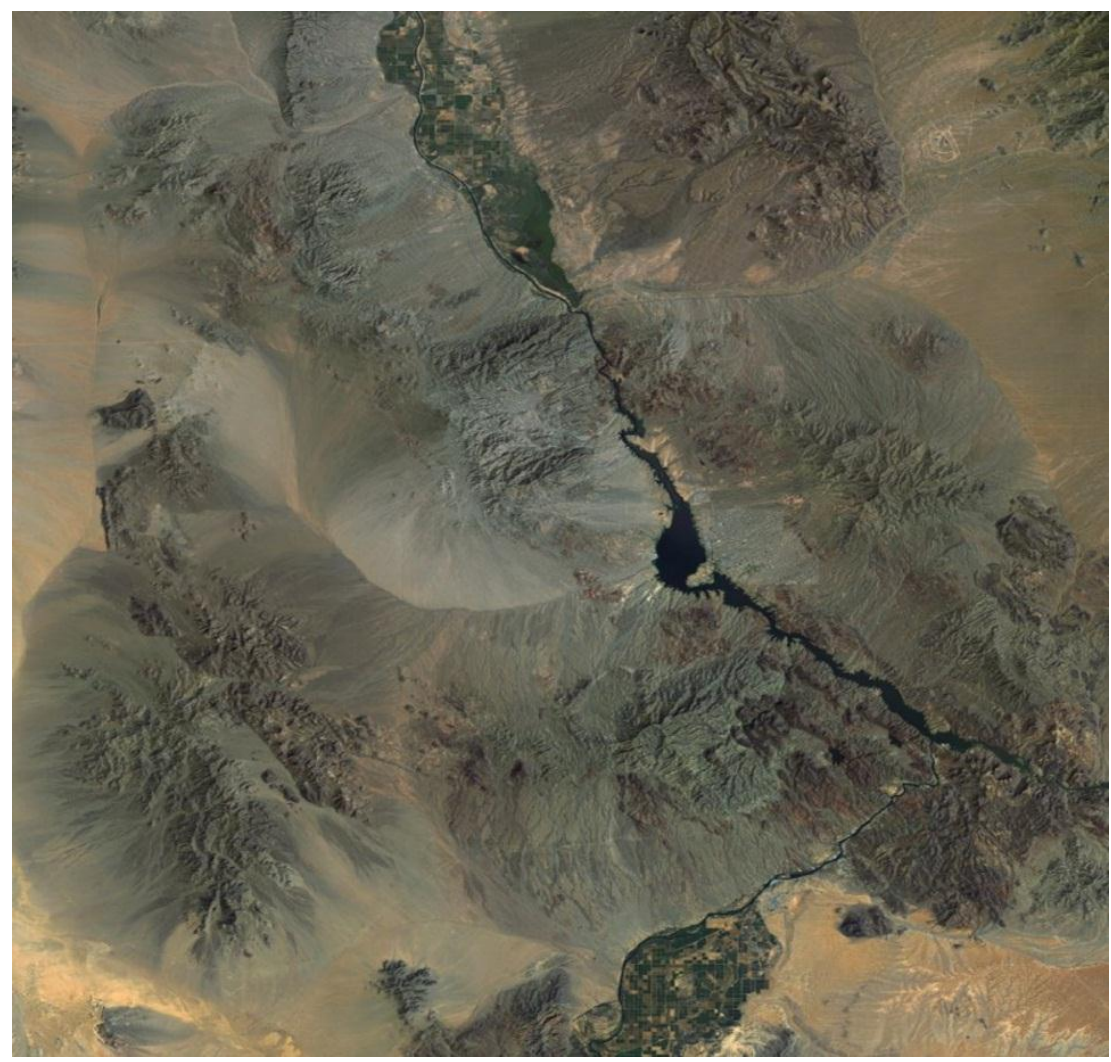

Figure 1-1: The study area.

\subsubsection{Methods}

The first step was to research reports and articles about search and rescue operations and other articles pertaining to lost person behavior. These articles were sought after to uncover common behaviors people exhibit when exposed to stressful situations and to provide insight on techniques to recreate these behaviors.

After discussion with the client, it was decided that the simulation would be created using the Python programming language because it allows for seamless integration with the ArcMap 10.0 environment.

The client's description specified that the tool acquire information as it travels and must not have "perfect knowledge of the entire scene... so that a script would indicate a 
more human approach to problem solving as it relates to finding one's way through the wilderness" (Christopher P., personal communication, April 17 $\left.7^{\text {th }}, 2012\right)$. The tool should restrict the environment similar to the way a person on the ground is restricted to what they can sense. Furthermore, the simulation must allow for a person to get turned around and "use a directional bias instead of a fixed end point, at least until the fixed endpoint is 'in range"" (Christopher P., personal communication, April 17 $\left.{ }^{\text {th }}, 2012\right)$. Meaning the tool should portray a traveler with an idea of their final destination, but the traveler does not know exactly where the destination is until it comes into view.

To create "a more human approach to problem solving," a cost surface was required to model human perception of environmental obstacles. For testing purposes, the cost surface was assigned values based on slope - the more level the terrain, the lower the travel cost. The program incorporated a visibility analysis to restrict the amount of information the program could utilize to simulate what a traveler would see. The program also included variables to account for direction and erratic behavior by altering cost surface values. Travel speed was accommodated with the implementation of a recalculation percentage which was used to simulate a person's reevaluation of his/her path. The program records simulation decisions to produce a single determined path.

\subsection{Audience}

Experienced GIS users are the targeted audience for this paper, people who are familiar with geographic concepts and mapping software such as Tobler's Law and ArcMap. The audience should also be familiar with computer logic and terms such as iterator, module, and function. It is not expected that the user or the audience know everything about the topics listed, as the report will detail tool functions and logical processes.

\subsection{Overview of the Rest of this Report}

Chapter Two details research used to develop a simulation model. Chapter Three presents the project design. Chapter Four discusses the aspects of the database design. Chapter Five describes the program implementation. Chapter Six conveys analysis of the program outputs. Finally, Chapter Seven provides a conclusion and a discussion of future simulation development. 



\section{Chapter 2 - Background and Literature Review}

The path prediction tool incorporated two fundamental geographic analysis techniques least cost path analysis (LCPA) and viewshed analysis (VSA) - both of which are used in a range of disciplines from environmental biology to engineering. However, no studies were found that incorporate VSA and LCPA into a dynamic path prediction tool. Because of this, information from different fields was synthesized to gain an understanding of the analysis processes and procedures. Section 2.1 begins by examining applications of LCPA; Section 2.2 investigated sources of error with VSA; Section 2.3 discusses elevation model considerations; and Section 2.4 concludes with a summary.

\subsection{Least Cost Path Analysis}

Many studies were performed using a GIS-based least cost analysis tool (Collischonn \& Pilar, 2000; Saha, Arora, Gupta, Virdi, \& Csaplovics, 2005; Walker \& Craighead, 1997); however, no studies were found utilizing a restricted LCPA in a dynamic analysis. This section describes how the LCPA algorithm works and three cases where the algorithm was used to derive a path.

LCPA relies on a cost surface. A cost surface is represented in raster format and defines the cost - or difficulty - of traversing a cell. Careful consideration is required when constructing the cost surface because it governs the simulation output. For example, if a user wants to simulate a person trying to avoid sun exposure, lower costs should be assigned to forested areas. Many elements can contribute to the cost of a cell. For example, a more comprehensive cost surface may incorporate elevation, road networks, buildings, and waterways. These individual data elements are then reclassified to a scale, weighted to reflect importance, and combined into a surface. The generated surface may contain values in terms of travel speed through a cell.

Bagli, Geneletti, and Orsi (2010) described in detail how the LCPA algorithm worked to identify the cheapest path from one point to another over a cost surface. LCPA is performed by generating an accumulated cost surface by calculating the accumulated cost of each cell, beginning from a starting point for the entire analysis area. The first step is to generate cost distance and backlink rasters using the Cost Distance tool within ArcMap. Beginning with the starting point, the algorithm searches among neighboring cells and selects the cell with the lowest value; this process is repeated until the destination is reached, then works in the opposite direction to create the backlink raster. The least cost path between the starting point and the destination is actually determined by working backwards from the destination point by choosing the lowest value cells.

Since the process is performed cell-by-cell, deriving the least cost path can take several minutes.

Lee and Stucky (1998) acknowledged that performing LCPA was not a new concept but with the development of faster and more powerful computers, the use of LCPA became more prevalent. To test the flexibility of the LCPA tool, Lee and Stucky created scenarios for three industries: environmental resource management, civil engineering, and military activities. For their purposes, they created unique friction equations for each of their scenarios to simulate different behaviors associated with each development. The authors planned hiking trails for the environmental resource management scenario, 
considering routes with low cumulative cost where cost was equated to physical effort and exposure. The result was a scenic path through the applied Digital Elevation Model (DEM) proving to be significantly longer than the control Euclidean path. The civil engineering scenario was intended to perform a least cost path for the construction of a pipeline. For this scenario, the cost surface was modified by removing restrictions on slope and increasing the preference for cover. The resulting path achieved minimum visibility but increased construction cost and distance in an effort to decrease environmental impact when compared with the control path. The last scenario simulated military reconnaissance, incorporating a cost surface preferring elevated but concealed paths. The goal was to see as much as possible without being seen. The resulting path achieved its goals when compared with the control path. Each scenario resulted in a different path based on the cost surface constructed, demonstrating the importance of the cost surface for path prediction.

A study performed by Delavar and Naghibi (2003) utilized LCPA for a civil engineering project. The goal of the study was to reduce the cost of construction for an Iranian oil pipeline. They considered pipeline length, topography, surface geology, river and wetland crossings, road and railroad crossings, and proximity to large population centers in their cost surface construction. High cost values were assigned to developed areas and sensitive environmental areas so the analysis would avoid these areas. The only restriction placed on the pipeline was the source and destination. The initial proposed pipeline was $34 \mathrm{~km}$ long; the new pipeline from the LCPA was $35 \mathrm{~km}$ long but $29 \%$ cheaper to construct. Like the study performed by Lee and Stucky (1998), the LCPA was unrestricted and the study had a heavy focus on cost surface construction.

Jobe and White (2009) performed a LCPA in the Great Smokey Mountains National Park to identify zones for permanent vegetation plots. The purpose of using the LCPA was to determine locations that hikers tend to avoid when they visit the park. The authors created their own hiking function in order to estimate energetic costs associated with traversing terrain with variant vegetation densities, which incorporated LCPA from caraccessible roads, friction associated with landscape features, and costs associated with inclination. Accessibility to areas was assessed and the product of the study was a list of locations suitable for permanent vegetation plots. This case is unique compared to Lee and Stucky (1998) and Delavar and Naghibi (2003) in the study looked for places people avoided, but again a comprehensive approach was used to determine cost surface values.

Each of these studies exemplify the range of LCPA applications; and through careful construction of the cost surface, unique behaviors are derived. However, it is important to note that each of these studies performed an unrestricted LCPA, meaning that each analysis had access to the entire study area. In the path prediction model proposed in this project, the LCPA was restricted to the visible area to simulate the dynamic decisionmaking process.

\subsection{Viewshed Analysis}

Viewshed is used to determine what can be seen from or at a location and is commonly used to determine desirable locations for unsightly structures such as radio towers. The viewshed algorithm calculates whether one point is visible from another by analyzing surrounding elevations for interference. However, there are problems identifying lines of sight and identifying obstructions. Interferences are interpolated by comparing raster cell 
elevations, making it important to use a high accuracy DEM. If a lower resolution DEM is used then large visible areas will be generalized. Research has been conducted on applications of and improvements to viewshed analysis (Riggs \& Dean, 2007; Le Yaouanc, Saux, \& Claramunt, 2010; Kim, Rana, \& Wise, 2004).

Riggs and Dean (2007) investigated causes for error in VSA tools. They investigated viewshed algorithms utilized by different software companies. In their experiment, they elected to use the raster-based elevation model because the "rasterbased viewshed analysis produced results that more closely mimicked field surveyed viewsheds than did TIN-based analysis" (Riggs \& Dean, 2007, p. 179). The authors noted other causes of errors extraneous to viewshed algorithms, which included floating point errors by computer processors and vegetative interferences. The issue of floating point number errors in computers has diminished as computers have become more advanced and are capable of handling more information. Dealing with vegetative interference is another issues requiring a new type of VSA that is not yet available. Their idea for handling vegetative interference was to produce a probabilistic viewshed instead of the widely produced binary viewshed. A probabilistic viewshed would incorporate visual permeability through vegetation and assign raster cells a value between zero and one. A probabilistic VSA would be an improvement over the binary viewshed if data were available. For their experiment, they progressed with the binary VSA. Their results found that no software package's VSA resulted in greater than $85 \%$ agreement with field observations when using a 4 meter resolution DEM. What they did find was that "ESRI software... produced results that more closely agreed with the field surveyed viewshed than any other software package" (p. 185).

It is important to emphasize the agreement between the observed viewshed and the best algorithm results: $85 \%$. Since the path prediction tool repeatedly calculated viewshed, errors could propagate throughout the simulation, creating an improbable result. It is important to use high quality data to minimize errors in viewshed calculations.

\subsection{DEM and TIN Considerations}

There are two primary elevation models: the Triangulated Irregular Network (TIN) and the DEM. There are advantages and disadvantages for each model.

TINs are based on triangular elements with vertices at sample points. Surface elements, or facets, consist of planes joining three adjacent points in a network and are constructed using Delaunay triangulation. TINs can easily incorporate discontinuities and may constitute efficient data structures because the density of triangles can be varied to match the roughness of the terrain (Kumler, 2006; Wilson \& Gallant, 2000). However, DEMs have emerged as the most widely used data structure because of their simplicity in computer implementation. DEM cell size will affect storage requirements, computational efficiency, and analysis result quality. For example, a 10x10 mile DEM requires less storage and is easier to process than a DEM with 10x10 foot resolution for the same study area. The disadvantage of DEMs is that they cannot easily handle abrupt changes in elevation and they often skip important details of the land surface in flat areas (Wilson \& Gallant, 2000). Several studies have been conducted comparing each elevation model, weighing the advantages and disadvantages (Wilson \& Gallant, 2000; Carlisle, 2005; Kumler, 1994; Rothley, 2005). 
Kumler (1994) compared efficiencies between TINs and DEMs by creating models to represent a variety of terrains. To make the comparison, the TINs were limited to contain one-tenth as many points as the corresponding DEMs to make sure disk space was approximately equal. One prominent conclusion from this study was that "the contour-based TINs developed... are more efficient than gridded DEMs at modeling terrains found in the United States" (p. 39). While Kumler maintains a preference for the visual appeal and efficiency of the TIN, he concludes that "...TINs are at best only as efficient as DEMs" (p. 41).

The path prediction tool utilizes the grid-format data structure for two reasons. First, when comparing VSA results, the TIN based analysis failed to produce an accurate result (Riggs \& Dean, 2007). Second, DEMs can be manipulated on a cell-by-cell basis, an attribute that proved important when verifying the VSA output.

\subsection{Summary}

Chapter 2 discussed the tools and DEM data format utilized within the simulation.

Section 2.1 evaluated uses of LCPA, and Section 2.2 conveyed research on VSA; the two major components of the path prediction tool. Section 2.3 reinforced the decision to use a grid-based elevation model. Chapter Three will describe the system analysis and design. 


\section{Chapter 3 - Systems Analysis and Design}

When creating a computer program, it is important to begin with a design prior to coding to save time and frustration. This project began with a general design of the entire program and, as the project progressed, detailed designs were thought-out and created for individual components. Section 3.1 restates the problem. Section 3.2 describes the project requirements separated into two categories: functional and non-functional. Section 3.3 discusses the overall system design, and Section 3.4 details the evolution of the project plan. Section 3.5 concludes with a summary.

\subsection{Problem Statement}

Without knowing the trends or paths people might utilize when traversing unfamiliar terrain, government agencies face great challenges in allocating resources efficiently and effectively to reach the targeted people. This type of situation occurs when people are lost during hiking or moving to avoid conflicts. Agencies need a tool to predict people's travel path given a set of parameters. The current path prediction method is to perform LCPA repeatedly in an attempt to reveal travel corridors. To better predict human travel, there needed to be a way to simulate a person's dynamic decision-making.

\subsection{Requirements Analysis}

Requirements are separated into two categories: functional and non-functional. Functional requirements pertain to elements included within the tool to produce the desired result. Non-functional requirements include the operational, technical, and transitional requirements needed to deliver and operate the program. Table 1 lists the requirements of the developed tool. 
Table 1. Functional and non-functional requirements.

\begin{tabular}{|c|c|c|}
\hline Category & Requirement & Description \\
\hline \multirow[t]{6}{*}{ Functional } & $\begin{array}{l}\text { Decision- } \\
\text { making }\end{array}$ & $\begin{array}{l}\text { The system models human decision making based } \\
\text { on user inputs. }\end{array}$ \\
\hline & $\begin{array}{l}\text { Determine a } \\
\text { travel path }\end{array}$ & $\begin{array}{l}\text { The system determines a travel path by iteratively } \\
\text { performing a restricted least cost path analysis, } \\
\text { and combining the results into one contiguous } \\
\text { path. }\end{array}$ \\
\hline & Observer height & $\begin{array}{l}\text { The system models observer height by adding a } \\
\text { height attribute to the current observer point. }\end{array}$ \\
\hline & Directional bias & $\begin{array}{l}\text { The system dynamically alters the cost surface to } \\
\text { influence the simulation towards a destination. }\end{array}$ \\
\hline & $\begin{array}{l}\text { Fail-safe } \\
\text { sequence }\end{array}$ & $\begin{array}{l}\text { The system has elements to ensure model } \\
\text { processes are performed correctly. }\end{array}$ \\
\hline & $\begin{array}{l}\text { Randomization } \\
\text { function }\end{array}$ & $\begin{array}{l}\text { The system has the ability to randomize a cost } \\
\text { surface input with user-defined values and } \\
\text { coverage percentage. }\end{array}$ \\
\hline \multirow[t]{7}{*}{$\begin{array}{l}\text { Non- } \\
\text { Functional }\end{array}$} & $\begin{array}{l}\text { Stand-alone } \\
\text { computing } \\
\text { environment }\end{array}$ & $\begin{array}{l}\text { The system operates independently of internet } \\
\text { and network connections. }\end{array}$ \\
\hline & ArcMap 10.0 & $\begin{array}{l}\text { The system was constructed using ArcMap } 10.0 \\
\text { tools and functionality. }\end{array}$ \\
\hline & Spatial Analyst & $\begin{array}{l}\text { The system utilized tools from the ArcMap } 10.0 \\
\text { Spatial analyst extension. }\end{array}$ \\
\hline & Python 2.7 & $\begin{array}{l}\text { The system was programmed with Python } 2.7 \text { for } \\
\text { seamless integration with the ArcMap } 10.0 \\
\text { environment. }\end{array}$ \\
\hline & User interface & $\begin{array}{l}\text { The simulation was linked to an ArcMap } 10.0 \\
\text { tool interface to facilitate the user experience. }\end{array}$ \\
\hline & Delivery & $\begin{array}{l}\text { The system was delivered via compact disc to the } \\
\text { client. }\end{array}$ \\
\hline & $\begin{array}{l}\text { Help } \\
\text { documentation }\end{array}$ & $\begin{array}{l}\text { The program contains help documentation and the } \\
\text { program code is annotated with descriptive } \\
\text { comments. }\end{array}$ \\
\hline
\end{tabular}

\subsubsection{Functional Requirements}

The model has several functional aspects, both to enable the simulation to operate and to enhance the model's final prediction. Overall, the tool models human travel over a terrain in a predetermined direction to simulate human decision making based on information perceived by an individual operating within an environment. To simulate the decisionmaking process, the model identifies the best route through the current visible area, stops 
at a predetermined percentage of the route, and reevaluates. This approach is used because the model assumes the individual has limited to no knowledge of the operational environment and no modern guidance tools, such as a handheld global positioning device, to aid in navigation.

The model makes the assumption that the person has a general heading. For example, if a person is traveling from Los Angeles to Baltimore, the general plan is to move east. To create this behavior, the simulation works towards a final destination with a directional bias. The directional bias simulates the behavior of a person with some sense of direction. In the Los Angeles to Baltimore example, the person is aware of his general direction and knows he will not make progress towards the destination if he travels north, south or west, so he will do what he can to travel east. If a situation is encountered when the eastbound direction is not possible or too difficult, the simulation allows the individual to diverge from their primary heading and turn around if necessary. Figure 3-1 demonstrates these behaviors. Comparing the orange path without the direction bias to the purple path with the directional bias, the differences became clear. The purple path took a more direct route, while the orange path wandered a little more. Also notice that even though the purple path utilized the directional bias, it was still able to turn around to find an easier path.

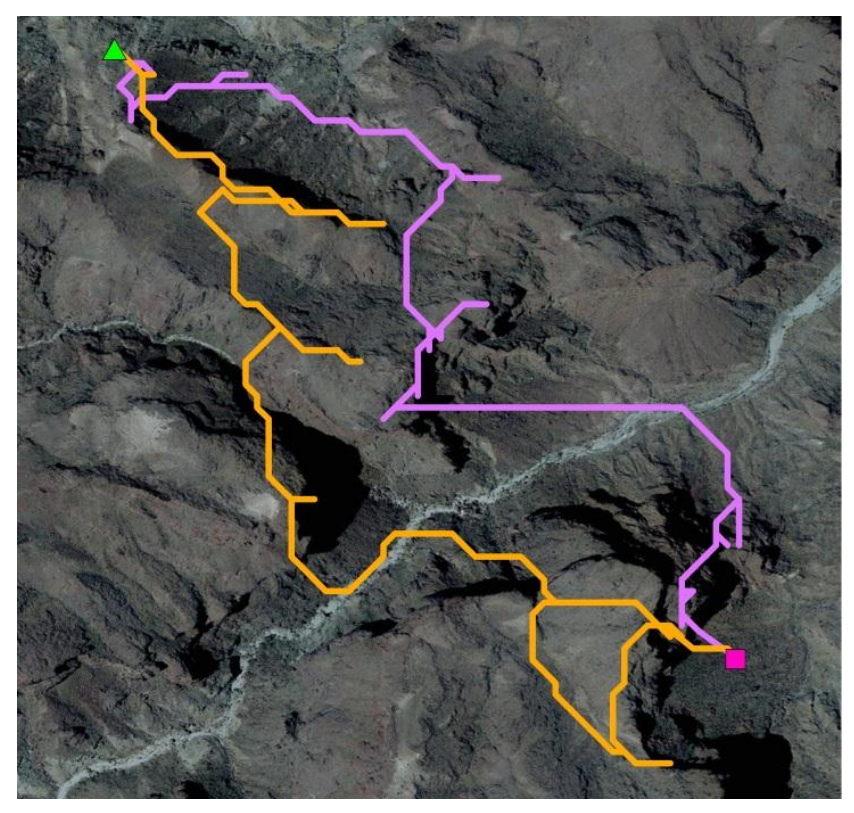

Figure 3-1: The influences of directional bias.

Observer height is an important parameter because it creates a more realistic visible area when appropriate height values are used. In instances when visibility is limited or non-existent, the model will increase the observer height by a factor of ten to increase visible area and force the simulation to progress. The increase in height is only temporary and can be equated to a person climbing a tree or some other object to survey the terrain. For the temporary height increase to occur, the model must first identify a traveler being immobile for a number of iterations. 
Built within the model is a cost surface randomization function. This function takes three inputs - percent cover, from, and to values - and uses these to randomize the cost surface. The randomization function takes points assigned with random values in the specified range and places them randomly on the cost surface, then adds or subtracts cell values based on the point value. The randomized cost surface does not alter the original cost surface file. The purpose of this requirement is to determine the importance of the determined path segments when the model is run repeatedly. If the cost surface is altered by five percent, locations where the path remains the same despite increased cost surface values are interpreted to have a higher probability of travel. Conversely, sections of the path can be interpreted as less important if the path changes due to surface alterations. If the model is run several times with a randomized cost surface; a user will notice the emergence of travel corridors.

\subsubsection{Non-Functional Requirements}

The model is designed for a stand-alone computing environment; no network connection is necessary as long as the required data are stored on a local drive. Furthermore, the user's machine must have ArcMap 10.0 with an ArcInfo level license, the Spatial Analyst extension, and Python 2.7.

When an analyst uses the tool, he/she is presented with a user interface similar to other ArcMap tools. However, a user must have knowledge of the study area and subjects in order to produce a reasonable result because there are model inputs that can be adjusted and can produce an unrealistic result. The most important aspect of the analysis is the creation of an accurate cost surface. The cost surface dictates how the travel expense is interpreted by the tool. To create a well-structured surface requires an understanding of the environmental impediments from a traveler's perspective.

When the program was delivered, it was accompanied with complete help documentation to facilitate user experience. Each data entry field within the user interface has a description of the file requirements or a description of the variable and its application. The program code contains comments so a non-Python user or nonprogrammer can easily read the comments and decipher what action the program will execute on each line or code block. All of the code, user interface, and help documentation were delivered via compact disk to the client.

\subsection{System Design}

The system design revolved around the functional and non-functional requirements and can be adapted for use in an ArcMap-based GIS. Figure 3-2 portrays the system design. Beginning from the left, the user is presented with a graphic user interface (GUI) where they will populate the optional and required fields. The data is then accessed by the program and the simulation begins. Upon completion, the program will have modified the starting point file and produced two output paths to a geodatabase. 


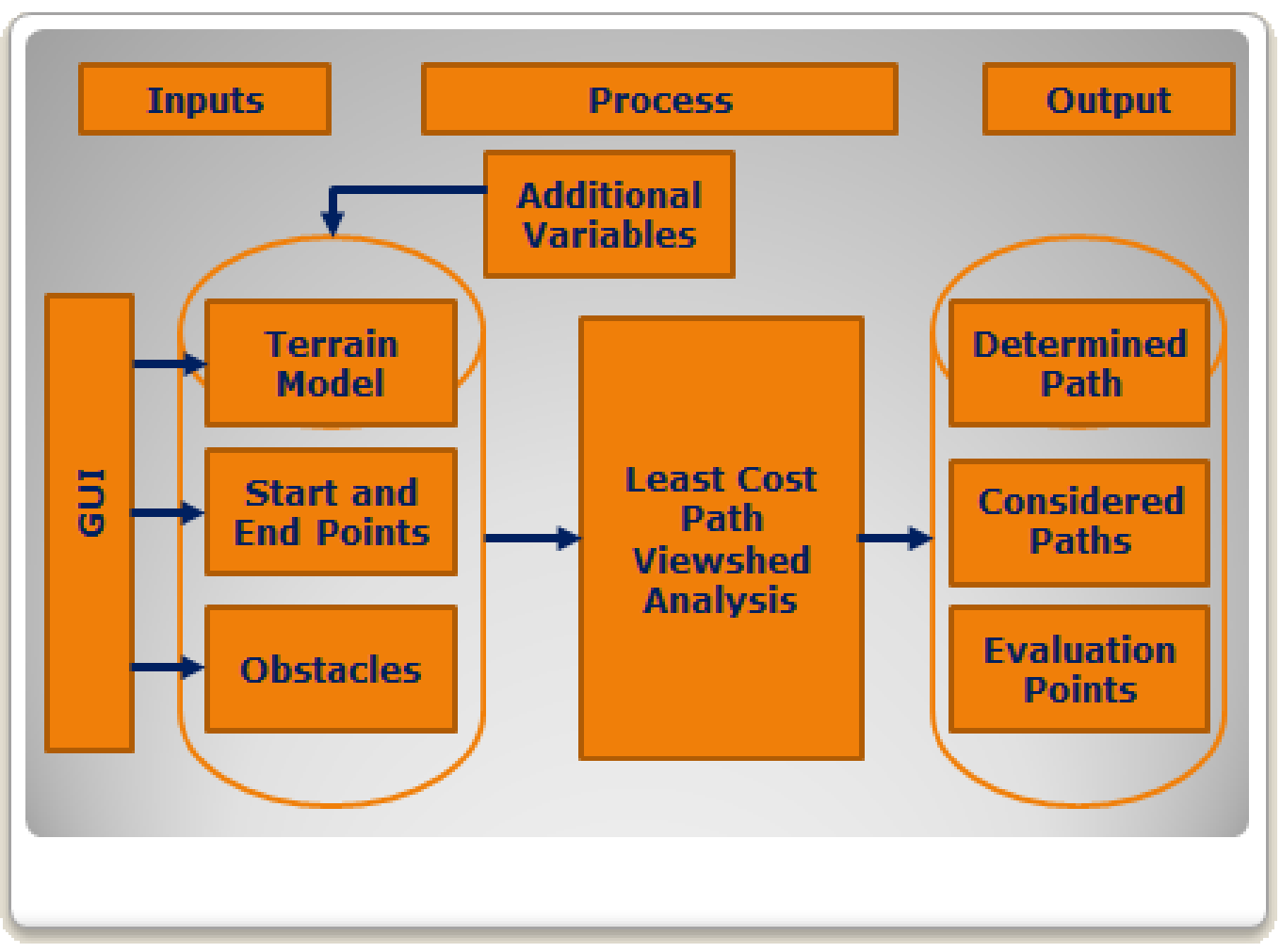

Figure 3-2: General system design.

\subsection{Project Plan}

When the project began, there was a lot of uncertainty with scheduling because the program development process was uncertain. It was suspected the building phase would require several weeks because skills and familiarity with the ArcMap module within Python had to be acquired. At the time, the build phase was divided into two components: the raster randomization tool and the simulation.

In the original schedule the design phase was allotted four days. In these four days the core functionality of the tool was decided; the simulation would only include the combined LCPA and VSA and work with the visible information until the final destination is reached.

Once the basic design was in place, work was started on a prototype, marking the beginning of the build phase. The build phase was allotted 64 days. With this amount of time a December 12 deadline was feasible.

Comparing the project plan from the beginning to the end, several aspects changed. First, the thought that designing, building, and testing were separate phases was unrealistic. Once the initial design was in place, coding was completed and tested, and the process was repeated to make improvements and additions. The linear waterfall approach depicted in Figure 3-3 shows the original project plan. 


\begin{tabular}{|c|c|c|c|c|c|c|c|c|c|c|c|c|}
\hline & Jan & Feb & Mar & April & May & June & July & Aug & Sept & Oct & Nov & Dec \\
\hline \multicolumn{13}{|l|}{ Proposal } \\
\hline \multicolumn{13}{|l|}{ Plan } \\
\hline \multicolumn{13}{|l|}{ Design } \\
\hline \multicolumn{13}{|l|}{ Build } \\
\hline \multicolumn{13}{|l|}{ Test } \\
\hline \multicolumn{13}{|l|}{ Document } \\
\hline \multicolumn{13}{|l|}{ Defend } \\
\hline Edits & & & & & & & & & & & & \\
\hline
\end{tabular}

\section{Figure 3-3: The project plan.}

The actual Design, Build, and Test phases are more accurately portrayed by Figure $3-4$, which displays the agile development approach. The agile approach allowed for rapid development because coding was completed, tested, and feedback was received for further improvements.

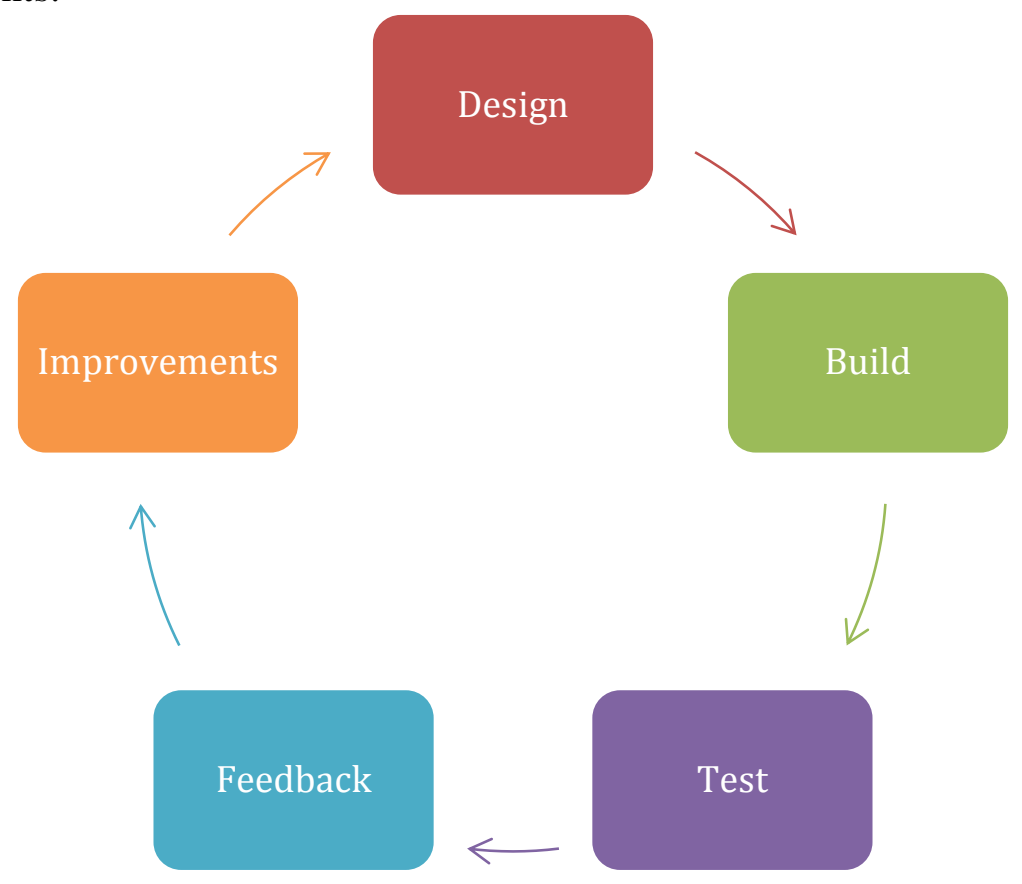

Figure 3-4: The agile development cycle.

Prior to constructing a project plan, steps were taken to identify possible risks. The two main risks were lack of experience with programming and project planning. To manage these risks, development started early and more than enough time for construction was scheduled. These were two necessary and appropriate actions for handling the identified risks and the project was completed with all desired functionality within the original time frame. 


\subsection{Summary}

This chapter described the considerations and plan development for project completion. The two lessons learned were to start early and allow for more than enough time to finish the project. A client will never be upset if a project is finished ahead of schedule. 



\section{Chapter 4 - Database Design}

A database was required to store the inputs and outputs of the program. This chapter describes the design and implementation of the database. Section 4.1 discusses aspects of the conceptual model and Section 4.2 details how the conceptual model was implemented. Section 4.3 identifies the source of the test data; Section 4.4 discusses additional test data collection; and Section 4.5 covers data preparation. Chapter Four concludes with a summary in Section 4.6.

\subsection{Conceptual Data Model}

The client needed a way to predict where people will travel through an environment. Conceptually, there are four components: the person, the environment, a location, and the route the person will follow.

In Figure 4-1, "Person" is connected to path, location, and surface because a person lives and travels across a surface and they must always have a location. Whenever a person moves, they create a path. There is always a location where the person has been and where they will be in the near future. A person makes decisions about where to travel based on the surface and how they interpret it based on what can be seen. For this reason a dotted line is used to connect person and surface. As mentioned, a person always has a location and multiple locations can be connected to reveal a path, thus a location is connected with path with the composition symbol (a black diamond).

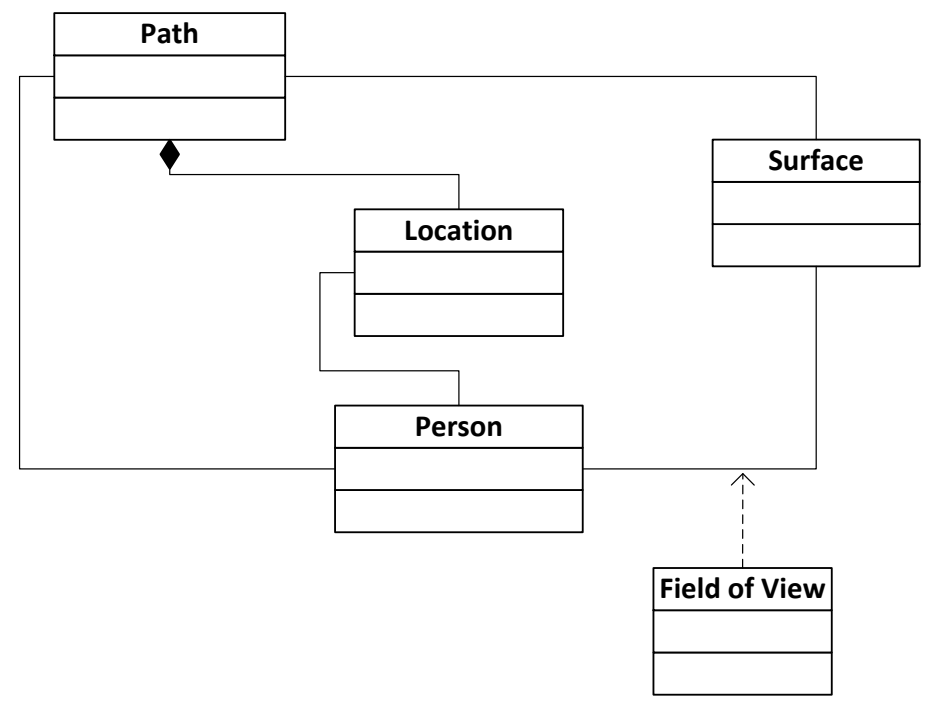

Figure 4-1: Conceptual model.

\subsection{Logical Data Model}

Each component from Figure 4-1 was generalized because it would be inefficient to incorporate all aspects of reality in a model. 
For the purpose of this simulation, Person was represented by a point. A person is a three dimensional object with many attributes, whereas a point is a zero dimensional object that has a location attribute. In the process of the simulation, a person is represented with a point with height attribute. Other aspects of human behavior were incorporated within the model but were not directly associated with the point feature.

Using the point feature with a height attribute and a digital elevation model (DEM), a field of view was calculated and represented with a binary surface of what can and cannot be seen from the perspective of the point. The binary result of the field of view was then draped over the cost surface to represent how a person interprets the environment.

The cost surface was not generated by the simulation but it was required to perform the analysis. A cost surface can be composed of many different elements to communicate how the environment is interpreted by the simulated person. Accurate cost surfaces include elements such as land cover and slope, and consider human characteristics like age and mental capacity. The analysis performed for this project used a cost surface based on slope; the steeper the slope the higher the cost. The cost surface was generated one time before the model was run. The model did modify the surface to create certain behaviors, including restricting the surface with the visible area.

The determined and considered paths were stored as lines with a length attribute. The paths were derived from raster data, meaning that accuracy for the determined and considered paths was only as spatially accurate as the raster data resolution. In no set order, the files were stored in the Geodatabase. Upon completion of the simulation, the Geodatabase is updated with two new line feature files - the predicted travel path and the considered path - and one updated point feature file containing the evaluation locations. During the analysis, intermediate raster files were stored within the database but were deleted when the analysis was complete.

\subsection{Data Sources}

Data for the project arrived in the form of a compact disc containing a single DEM. The DEM covered an area of the Southwestern US including parts of southern California, southern Nevada, and western Arizona. Other required elements were generated from the DEM.

\subsection{Data Collection Methods}

Additional data were collected for model tests from the United States Geological Survey (USGS) Seamless Viewer web portal. Using the USGS web portal data were extracted for an area covering parts of western Maryland, southern Pennsylvania, northeast West Virginia, and northern Virginia.

\subsection{Data Scrubbing and Loading}

It was discovered through model testing that there was one major inhibiting factor of DEMs that had to be handled prior to analysis: DEMs must be filled. This step is important because DEMs contain errors where elevation values are significantly lower than surrounding cells. The ArcMap Spatial Analyst tool Fill was used to locate the sinks 
and correct their elevation values. Without performing this step, the point representing the person can fall into a depression and get stuck, and the analysis will not be able to continue. The requirement to fill the DEM is explicitly stated in the tool help window in the interface, in the tool metadata, and was explained to the client.

\subsection{Summary}

This chapter included elements of the conceptual model and how it was translated into the computing environment. This chapter also discussed the source of testing data and how they were prepared for use. Chapter Five will detail how the model was implemented in a system. 



\section{Chapter 5 - Implementation}

Chapter 5 describes in detail the steps used for creating the model and the logical flow of the program. Section 5.1 begins with the initial program layout. Section 5.2 specifies the required program modules, variable declaration, and environment settings. Section 5.3 describes the three assembled functions and their use. Section 5.4 explains the initial input modifications. Section 5.5 shows how the model dynamically calculates a restricted least cost path, and Section 5.6 describes the simulation completion and clean-up.

\subsection{Model Builder}

The first step was to build a working model overview. To do this, Model Builder was implemented and tools were linked using graphic representations. Model Builder provides the convenience of a quick initial design - tools are combined and workflows are constructed using the drag-and-drop capability and are displayed graphically. Model Builder verifies workflows and inputs, and identifies problematic linkages. Figure 5-1 displays a high-level overview of the program abstracted from the Model Builder diagram. It begins at the upper-left corner and progresses towards the bottom-right corner.

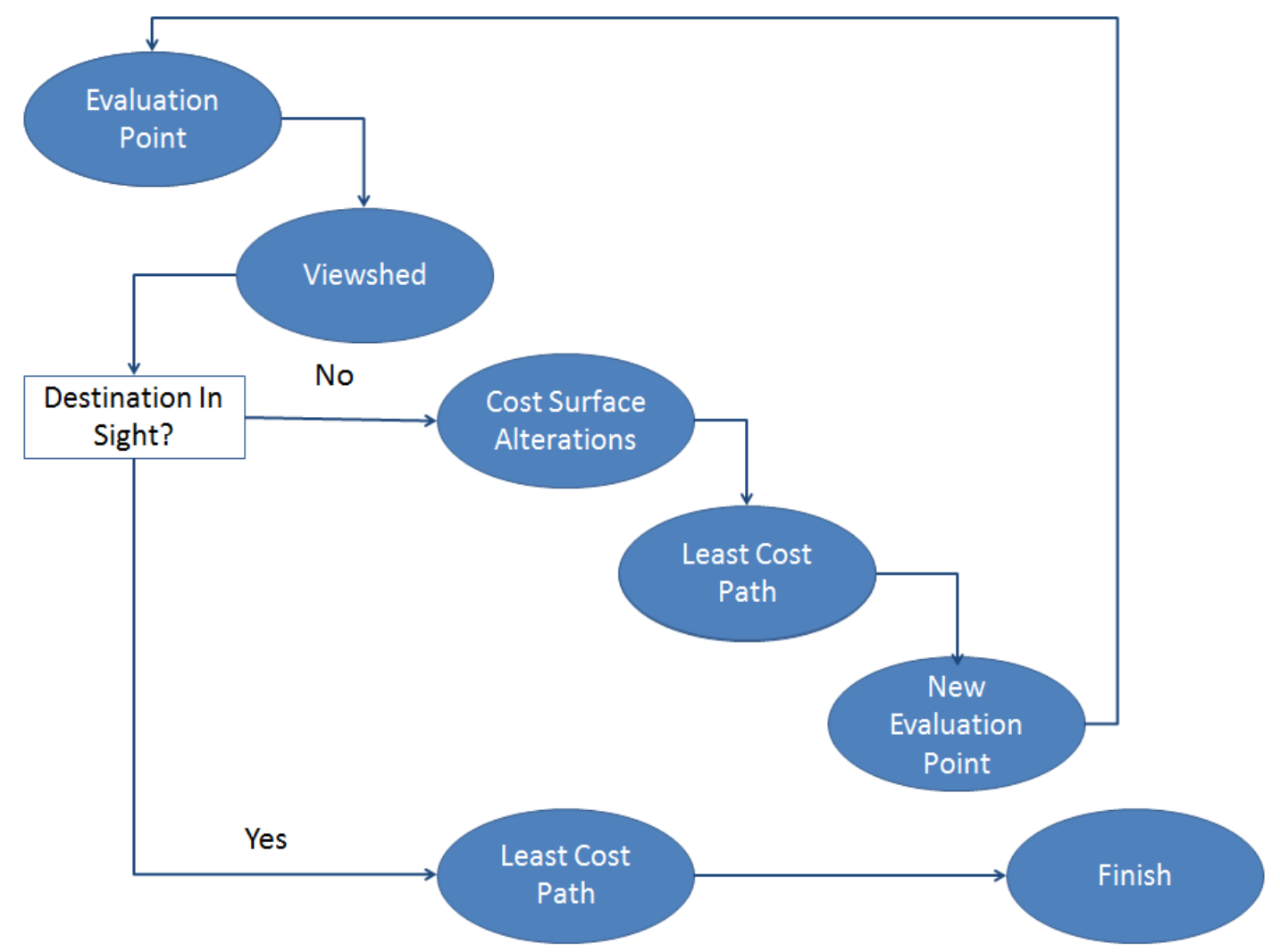

Figure 5-1: Program overview. 


\subsection{Modules, Variables, and Environments}

After completing the Model Builder workflow, the next step was to export the model as a Python script and import the additional required Python modules. When a module is imported, Python exposes its functional services for use in a script. The modules used for this simulation included arcpy, random, math, os, and numpy. The arcpy module allows for the use of ArcMap tools within Python; the random module is used in the raster randomization function; the math module is used to access high-level functions such as arctangent; os is used for operating system commands; and numpy is used for matrix and array manipulation. An overview of the completed program is presented in Figure 5-2. The figure displays the program in more detail, and the remainder of the chapter will follow the flow of this diagram. 


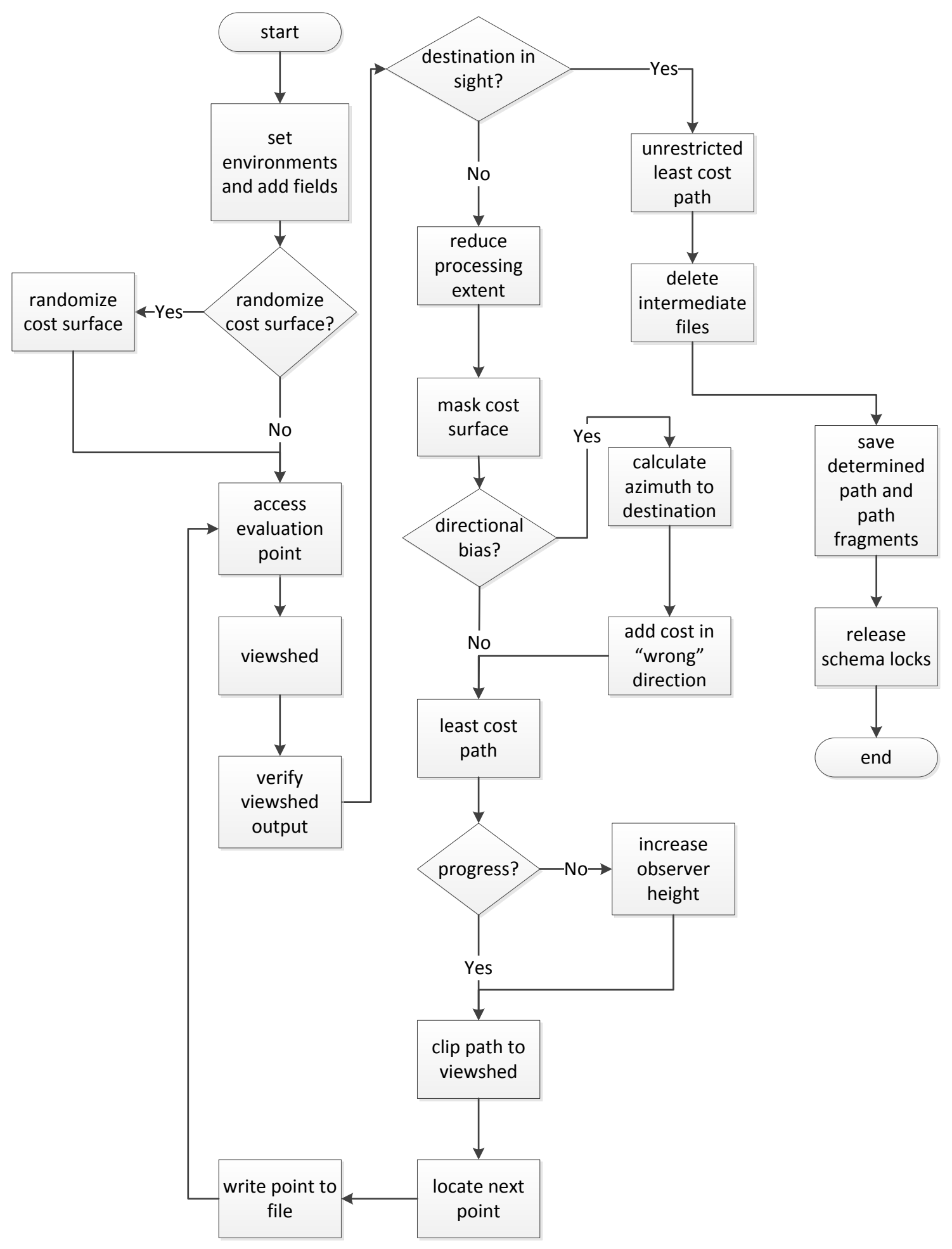

Figure 5-2: The program diagram. 
Because the simulation uses tools from the Spatial Analyst toolbox extension, it is important for the script to first check if the machine is licensed to use these tools. Otherwise the program will report an unexpected error.

The model takes a maximum of 17 variables - six are required and 11 are optional. Each variable is given a unique and descriptive variable name in the script for code readability. The order of the variables is not important because they were each assigned a unique index for linkage to the user interface.

The "percent cover" parameter (used for raster randomization), and the "lost radius" parameter (used to determine progress) are both taken as integers so a user is not required to make calculations or conversions. Percent cover is converted from a whole number to a decimal; for example, an input of 70 is converted to 0.7 . The lost radius parameter is in the unit of cell. Spatial resolution from the input raster must be retrieved and multiplied by the lost radius unit. For example, if a user enters a value of ten for the lost radius and the width of each cell is two feet, the program will convert the lost radius to 20 feet. Both of these conversions are performed within the code to facilitate the user experience.

With the parameters in place, it is important to set the workspace and environment settings. These settings can be specified manually through ArcMap menus; but to provide a smooth user-experience, these settings are derived from the model inputs and defined in the code. Without setting these parameters, the program will not know where to store intermediate files or what coordinate system to use.

\subsection{Functions}

Functions were created within a model. Using functions are similar to using modules but these modules must be created. Table 2 outlines each of the functions created for the simulation.

Table 2. The program functions

\begin{tabular}{|l|l|l|l|}
\hline Function & Inputs & Output & Description \\
\hline DestInView & $\begin{array}{l}\text { destination point, } \\
\text { viewshed }\end{array}$ & True or False & $\begin{array}{l}\text { This function } \\
\text { determines if the } \\
\text { destination can be seen } \\
\text { and signifies the } \\
\text { program to conclude. }\end{array}$ \\
\hline randomRaster & $\begin{array}{l}\text { workspace, } \\
\text { reference raster, } \\
\text { percentage cover, } \\
\text { from range, to range }\end{array}$ & $\begin{array}{l}\text { A randomized } \\
\text { raster }\end{array}$ & $\begin{array}{l}\text { This function is used to } \\
\text { create a random raster } \\
\text { surface to be combined } \\
\text { with the input cost } \\
\text { surface. }\end{array}$ \\
\hline BreakOut & $\begin{array}{l}\text { evaluation points, } \\
\text { current evaluation } \\
\text { point ID, lost radius }\end{array}$ & $\begin{array}{l}\text { Increased observer } \\
\text { height }\end{array}$ & $\begin{array}{l}\text { This function is used to } \\
\text { help the program } \\
\text { progress if the } \\
\text { simulation gets stuck. }\end{array}$ \\
\hline
\end{tabular}


The first function is "DestInView" and takes the destination point and viewshed as parameters. DestInView determines when the simulated person can see the destination. To accomplish this, a spatial join is performed on the viewshed and the destination. The spatial join is set to join only objects that are completely within the viewshed. If the destination is within the viewshed, a field within the spatially joined table will be assigned a value of one; if the destination is not within the viewshed the field will be assigned a value of zero. When the destination is within sight, the function reports this information and signals the end of the simulation. If the destination is not within sight, the simulation's iterative process continues. Figure 5-3 displays how the DestInView function works. The blue stars are evaluation points, the blue triangles are the destinations, and the orange polygons represent the visible area. The diagram on the right shows an instance in which the function would continue with the iterative simulation; and the diagram on the left shows an instance in which the destination is within sight and the simulation would conclude.
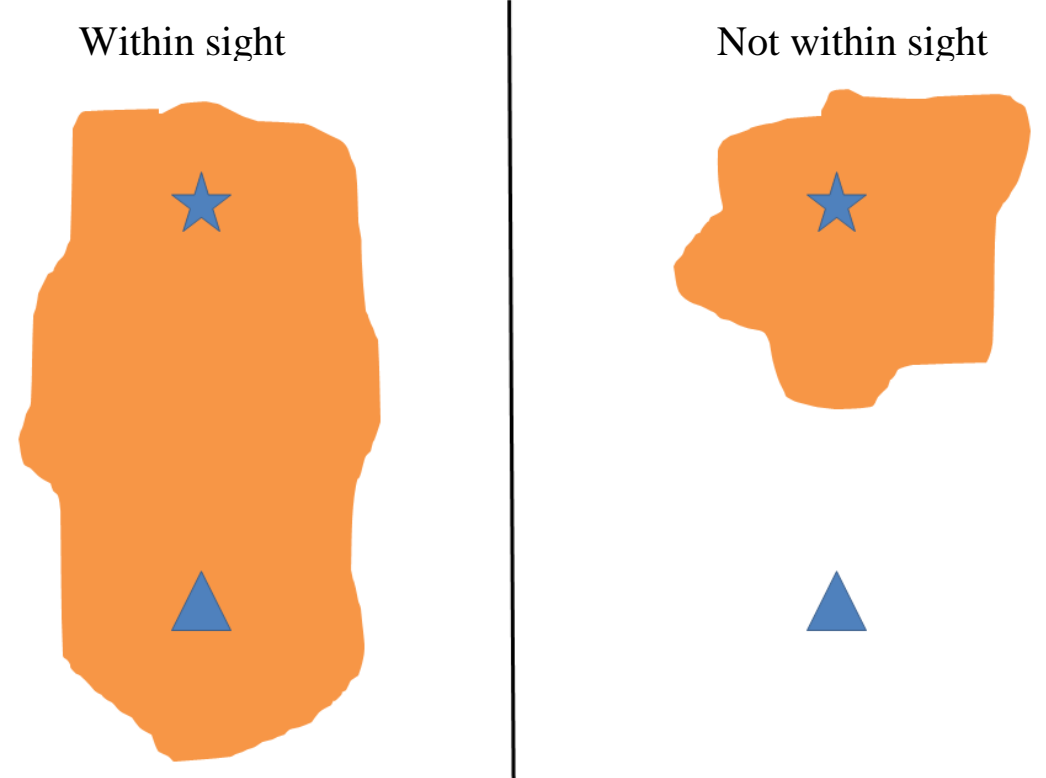

Figure 5-3: DestInView function.

The next function is "randomRaster" and takes the workspace, a reference raster, percentage cover, from range, and to range as parameters. The randomRaster function is used to create the randomness in the cost surface. This function retrieves information from the reference raster including spatial extent, cell size, and coordinate system. It then creates a table that will be populated with random $\mathrm{x}$ and $\mathrm{y}$ coordinates within the extent of the reference raster. Each new file is assigned a random value based on the "from" and "to" range parameters. The table is converted to a point feature. The point layer is then converted to raster. Figure 5-4 displays the cost surface before randomization on the left, 
the randomized surface in the middle, and the randomized cost surface on the right. The table and point feature are deleted and the raster is returned to the simulation to be combined with the cost surface.
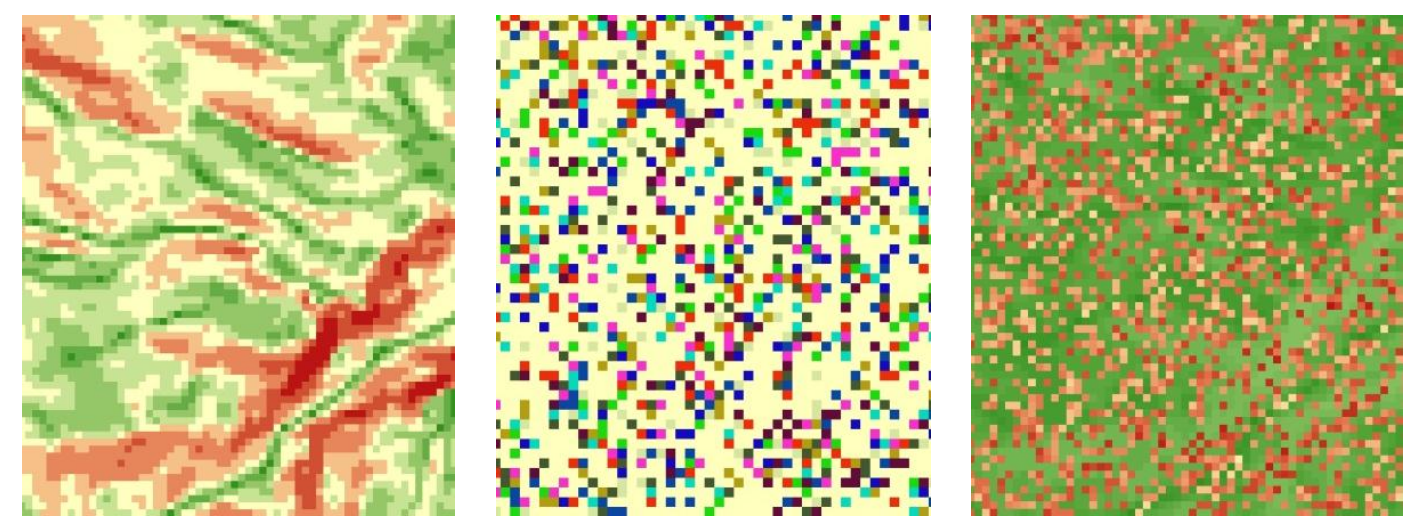

Figure 5-4: Random raster output.

The next function is "BreakOut" and takes the points feature class, the unique identifier of the current evaluation point, and the lost radius as parameters. The function then counts the number of previous evaluation points within the search radius. If the number of previous points exceeds the threshold set by a user, it indicates the person is stuck. The observer height for the following evaluation point will be increased to force the traveler to move out of the area.

\subsection{Initial Input Modifications}

With the functions defined, the code adds height information to the starting point feature class. A special field is added to the starting point feature class to communicate the number of units to offset the evaluation point from the surface of the DEM to the Viewshed Analysis (VSA) tool. It is important to know the units used for the DEM elevation value because these same units are used for the offset. If the DEM contains elevations in feet, an offset of six will be six feet.

The arcpy module contains cursors - insert, search, and update - used to read through feature classes row by row. A search cursor allows a program to read a file, an insert cursor allows for the addition of new records, and an update cursor is used to update a record. A cursor placed on a file creates a lock and can only be read by the program until the cursor is deleted. However, multiple cursors can be applied to a single file at the same time. Prior to the iteration, an insert cursor is applied to the starting point feature class. This is done prior to entering the iterator so the insert cursor is only opened once and remains open throughout the simulation. This cursor will be utilized later in the simulation.

At this point, the code will randomize the cost surface if the user has opted to do so. If not, the code will not randomize the cost surface and proceed. 


\subsection{The Iterative Process}

In addition to the insert cursor, a search cursor is opened on the starting point feature class. The search cursor is used to read and retrieve information from the evaluation point feature class and is used to start iterating through the evaluation points. A "for loop" is used for this model. A typical for loop will iterate through the number of items in a list. For example, if a list contains ten items, the for loop will iterate ten times. However, in the simulation, the list increases with each iteration. This is by design; code for exiting the loop is discussed later.

Model runtime varies depending on the size of the study area. To give the user some perception of progress, the model calculates the Euclidean distance from its current position to the destination point and then reports the progress as a percentage. If the model moves farther from the destination, the code will report that the simulated person has moved farther from the destination. The purpose of this is to avoid reporting negative percentages.

Next, viewshed is calculated and the visible area is extracted. Figure 5-5 presents a perspective view of the terrain utilizing in a 3D environment, however, viewshed is calculated in a 2D environment. Viewshed is calculated using a built-in function, resulting in a binary surface symbolizing the visible area and non-visible areas. The visible areas were then converted to a polygon feature. However, it was found that the VSA function had trouble converting the vector evaluation point to a raster for VSA. In some circumstances, the viewshed tool offsets the converted point by one cell and caused the program to fail. To account for this error, the program checks the conversion by changing the viewshed raster to a matrix and converting the point coordinates to row and column values. If the program finds the VSA has incorrectly calculated the evaluation point cell to be non-visible, it will correct this. Then the program will check if the destination is visible from the current evaluation point, if so the program stops iterating and exits the "for loop." 


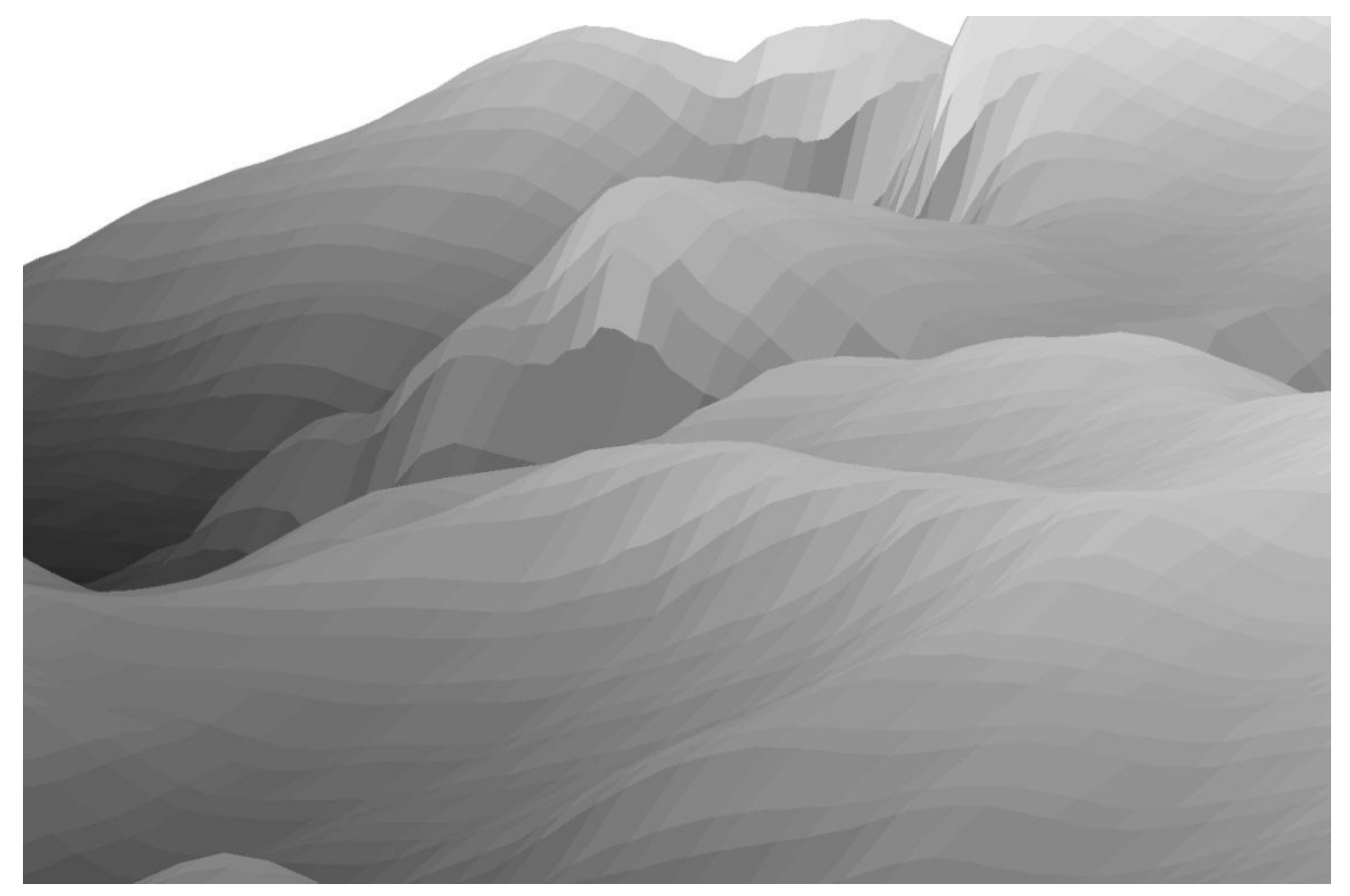

Figure 5-5: A perspective view of the terrain.

To decrease model runtime without sacrificing analysis, a dynamic analysis mask was created. The analysis mask restricts the processing extent of the geoprocessing tools to the masked area. To minimize the processing extent for the least cost path analysis, the current evaluation point, destination point, and computed viewshed are merged into a single feature class. From this single feature class, the program will derive the minimum bounding geometry of the features. The minimum bounding geometry of a feature is the smallest polygon encapsulating all features as shown in Figure 5-6. In the figure, the purple polygons and the two points are encapsulated by the blue polygon. The blue polygon is the minimum bounding geometry. The minimum bounding geometry has a smaller extent than the study area and is used to calculate the least cost path to reduce the number of cells for processing. At the beginning of each iteration, the processing extent is reset to the extent of the study area so the next viewshed calculation is unrestricted. All of the intermediate data for this process are stored temporarily in memory, decreasing the overall processing time for each iteration. 


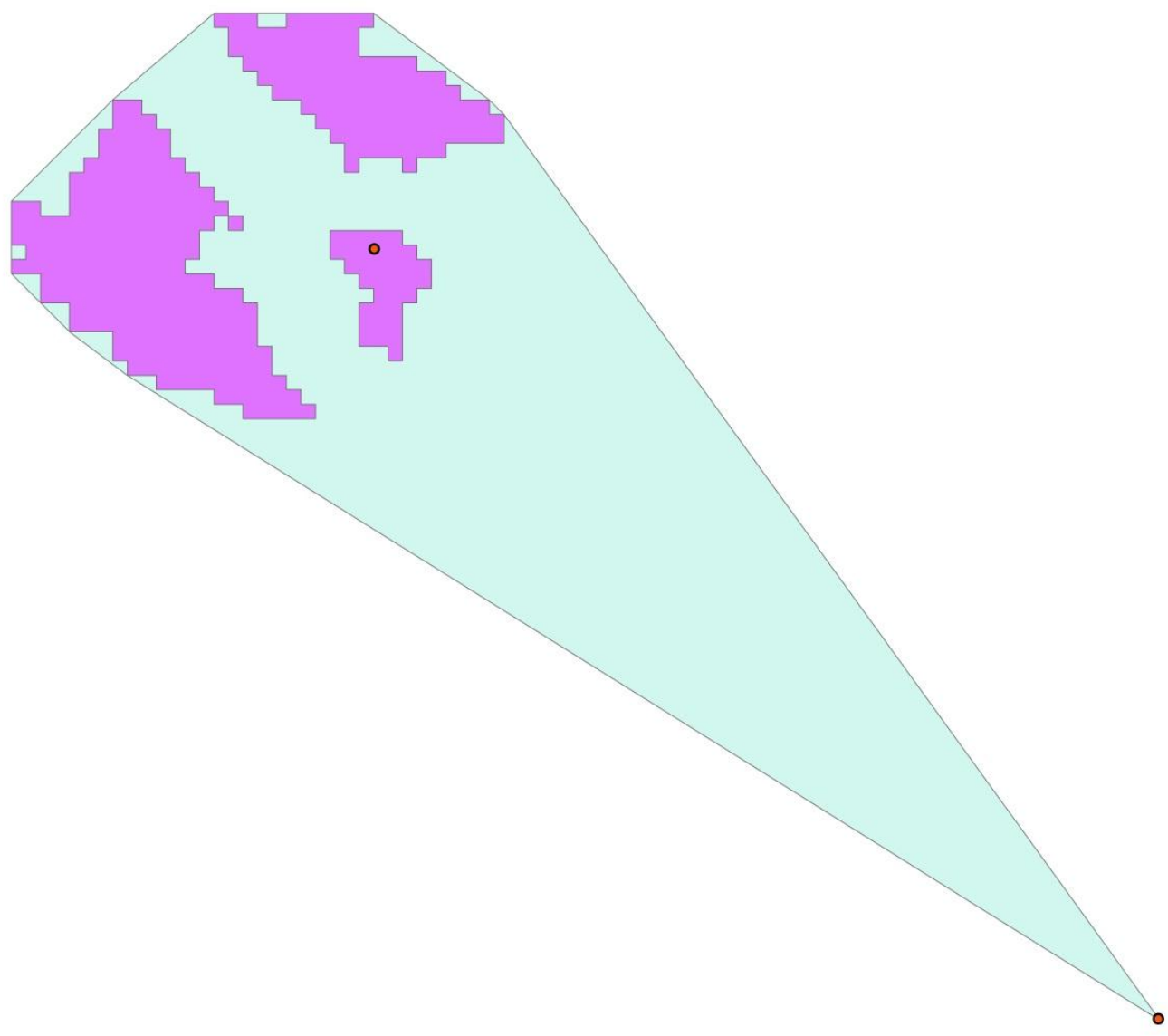

\section{Figure 5-6: Minimum bounding geometry for LCPA.}

To enforce traveling directions, a directional bias surface is created. The azimuth from an evaluation point to the destination is calculated. Preferred directions are those within a certain deviation to the calculated azimuth. The deviation is provided by a user; for example, with a value of ten and the azimuth from an evaluation point to the destination is $60^{\circ}$, the preferred directions are from $55^{\circ}$ to $65^{\circ}$. The original cost surface is modified by adding a constant cost to locations outside of the preferred direction. The added constant value is also provided by a user. Figure 5-7 is an example of directional bias added to a cost surface. In the figure, the red area of the surface has an increased cost, but the green area remains unchanged. The red area has an increased cost, but still retains the variation of the original cost surface and does not restrict travel through these areas. If no directional bias value is entered, the model will skip this step and continue with the original cost surface. At the start of each iteration, the original cost surface is used. The directional bias calculation is an intermediate process. 


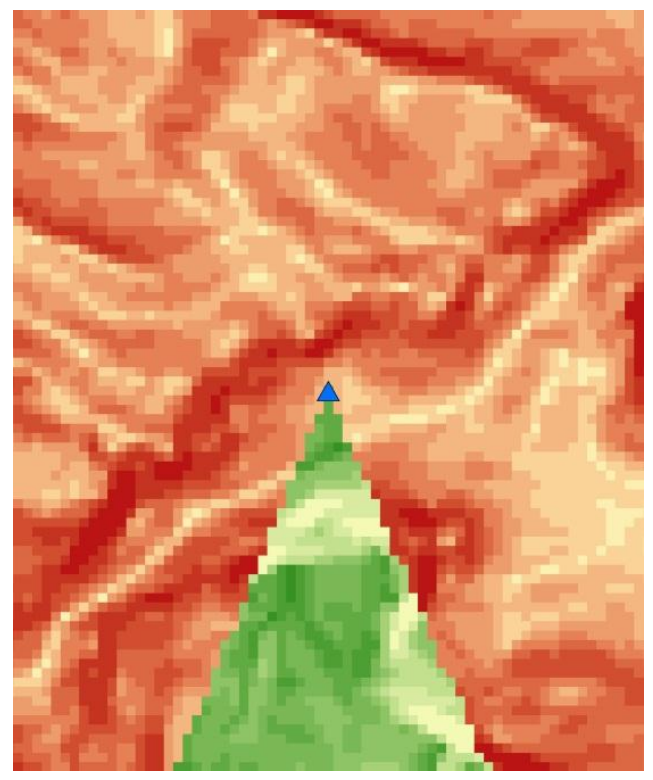

Figure 5-7: Southbound directional bias output.

Whether the cost surface is modified with a directional bias or not, the visible area of the cost surface is extracted. This is accomplished using the Extract By Mask tool, with the cost surface and viewshed result as inputs. This tool works like a clip where the cost surface is clipped with the viewshed. The result of the extraction is a subsection of the cost surface mirroring the visible area. The clipped cost surface now contains many cells with null values. These cells must be assigned a value for processing. To assign values to the null cells, a tool is used to perform an "if else" operation on the extracted cost raster surface to assign the null values with a user-specified high value indicating high risk to travel through the invisible area. Figure 5-8 is the final result of the cost surface restriction. The gray area was determined to be non-visible so it was assigned a value determined by a user. 


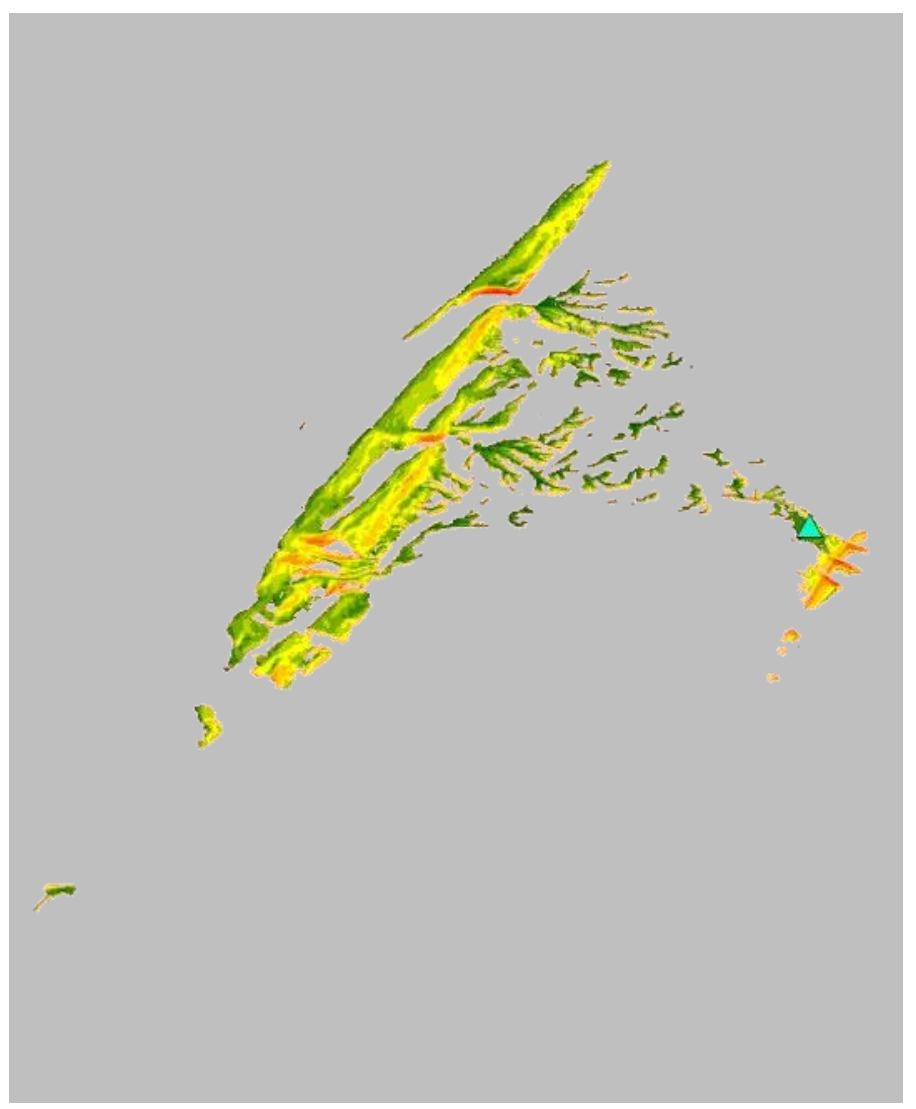

\section{Figure 5-8: A viewshed-restricted cost surface.}

With the cost surface prepared, the next step is to perform the LCPA. LCPA requires the calculation of a cost distance raster and calculates the accumulated cost from the evaluation point to the destination. The Cost Distance tool also creates a "backlink" raster, which contains the accumulated value back to the evaluation point. Both the cost distance and backlink rasters are then used for the LCPA calculation. The LCPA results in a path in raster format. The determined path is then clipped to the visible extent using the viewshed surface as the clipping layer. The determined path through the non-visible area was not useful because the cost surface values were changed. The clipped determined path is then converted from raster to vector format.

Now that the program has produced a partial path it must be saved to a file. Because the program is required to output both the determined path and considered path, two feature classes are created and the results are appended to the appropriate file.

After the data are stored, the program checks whether the simulated person makes progress. If the person has made little or no progress, the traveler's observer height will be temporarily increased by a factor of ten. This value is hard-coded in the program to reduce the number of inputs required.

The model will now progress down the determined path. The first step is to search for the closest line segment to the evaluation point. The closest line segment is selected and appended to the determined path output feature class. With the path stored the length of the line and the end point coordinates are retrieved to determine how far and in what 
direction the simulated person will travel on the path. The distance the person will travel is based on a percentage entered by a user such as $70 \%$ of the determined path. The distance to travel is determined by multiplying the user-defined percentage by the length of the line. For example, if the length of the line is ten units and the travel percentage is $70 \%$ then the next evaluation point will be seven units along the line. To generate the $\mathrm{x}, \mathrm{y}$ coordinates of the next point; the main line segment is split at each vertex into sub line segments. The program then locates the segment containing the new point. The coordinates of the line's endpoints are then retrieved and the new point is interpolated using the following equations:

$$
\begin{aligned}
& x=x_{A}+\left(x_{B}-x_{A}\right) * k \\
& y=y_{A}+\left(y_{B}-y_{A}\right) * k
\end{aligned}
$$

Figure 5-9 illustrates this process for the path in red. The program determined that the next evaluation point at $70 \%$ of the red line lies between points A and B. Segment AB is retrieved and the new point is interpolated using the equations above.

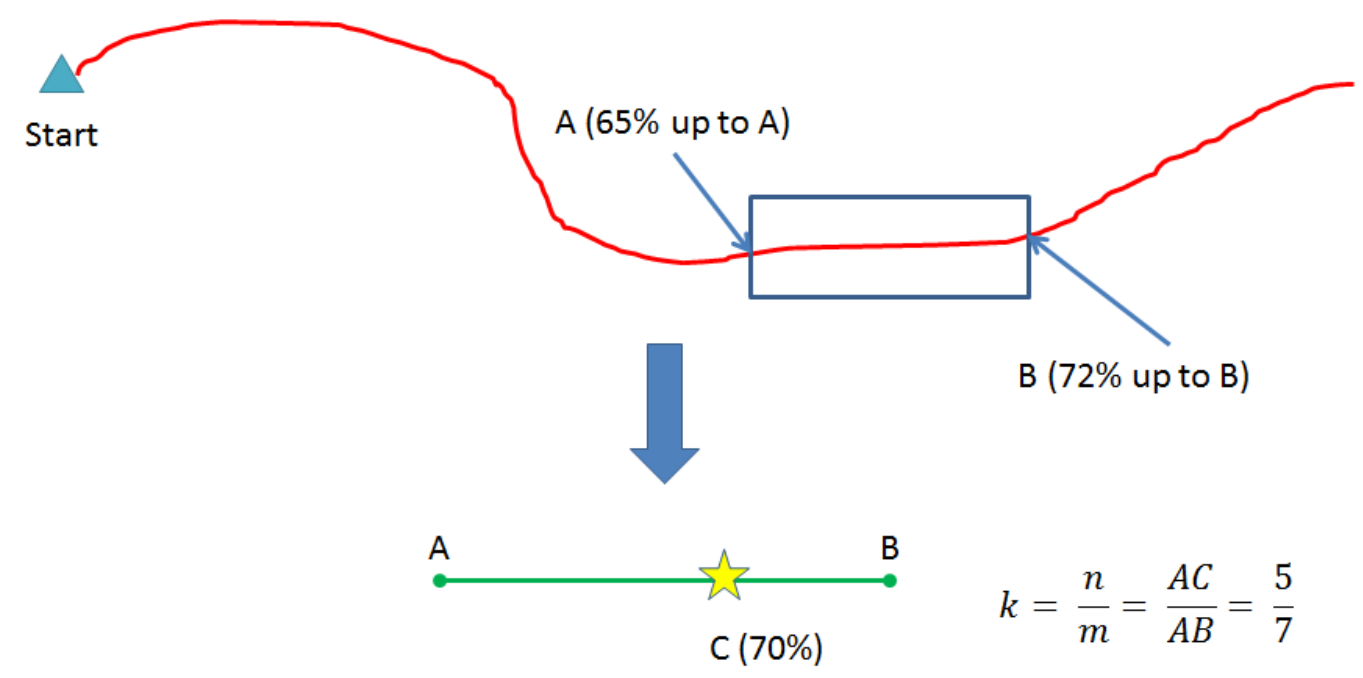

Figure 5-9: Determining the next recalculation point.

Once the coordinates of the next evaluation point are determined, the code writes the geometry of this point to the original starting point feature class. If the program determined earlier that the traveler made little or no progress, then the observer height will be modified for the new point at this time. Progressing from one to four, Figure 5-10 illustrates how the program predicts and then travels the path, and even turns around if necessary. The lighter lines were previously traveled and the darker, orange lines are the current route. 

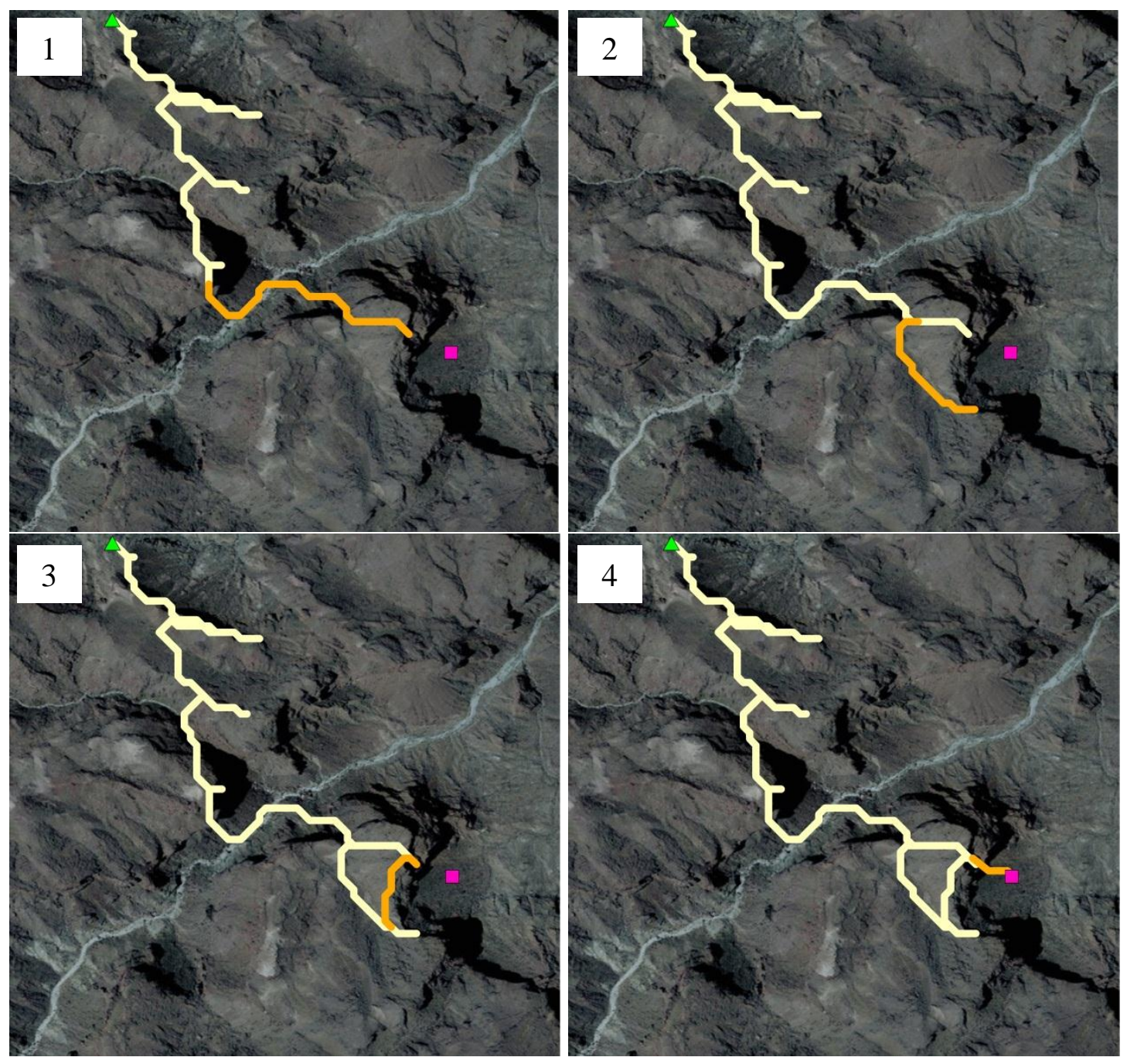

Figure 5-10: Traveling the path.

If the user wants to use a recalculation percentage of 100, however, the program will utilize a different sequence. Instead of segmenting the line to determine the next evaluation point, the code determines the travel direction and then copies and stores the end point for use in the next iteration.

\subsection{Simulation Completion}

If the program determines the destination to be within the sight, the program will exit the iteration and will perform a final LCPA using the last evaluation point and append the determined path to the output feature class. All open cursors and intermediate files are deleted and the program is complete. An example of the final model output is presented in Figure 5-11. The triangle is the starting point, the orange line is the determined path, the blue lines are the path fragments, the green dots are evaluation points, and the purple square is the destination. In the figure, the blues lines are mostly overlapped by the orange lines; however, the visible fragments are designated as considered paths. These 
paths were calculated through discontinuous portions of a viewshed analysis, which is why they are not part of the orange line.

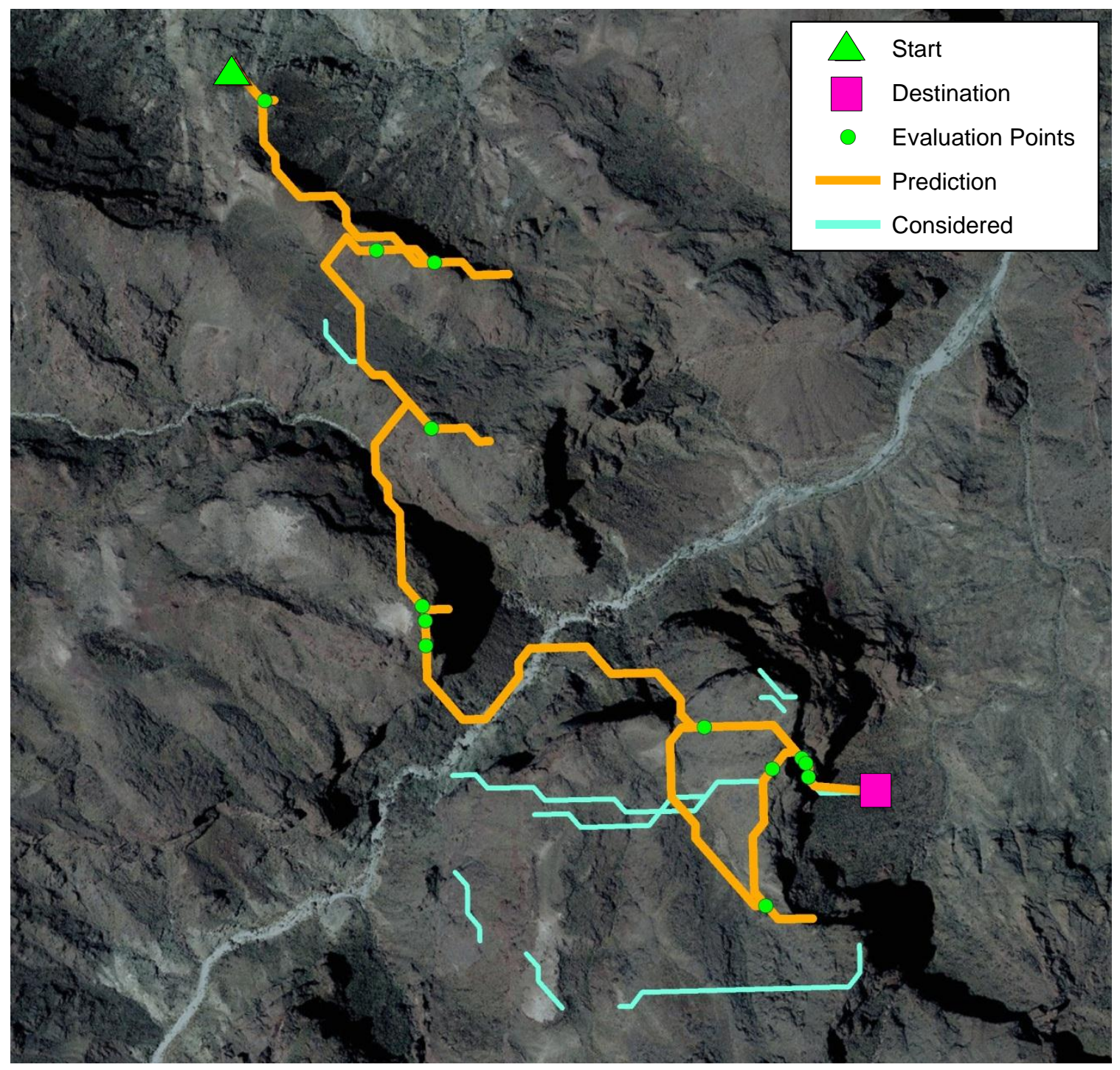

Figure 5-11: Simulation output. 


\section{Chapter 6 - Results and Analysis}

There are several elements that can affect the result of the path prediction tool. In this section, analysis was performed to determine the effects of variable changes. The control simulation used a DEM, a cost surface based on slope, $80 \%$ recalculation distance, and a two meter observer height. Section 6.1 compares the control simulation (Figure 6-1) on two DEMs to observe the differences between a rough terrain and smoother terrain. Section 6.2 examines the simulation changes from different recalculation percentages. Section 6.3 examines the effects of directional bias. Section 6.4 compares the simulation to a standard LCPA. Section 6.5 examines the effects of observer height. Section 6.6 discusses viewshed analysis error. Chapter 6 concludes with a summary in Section 6.7.

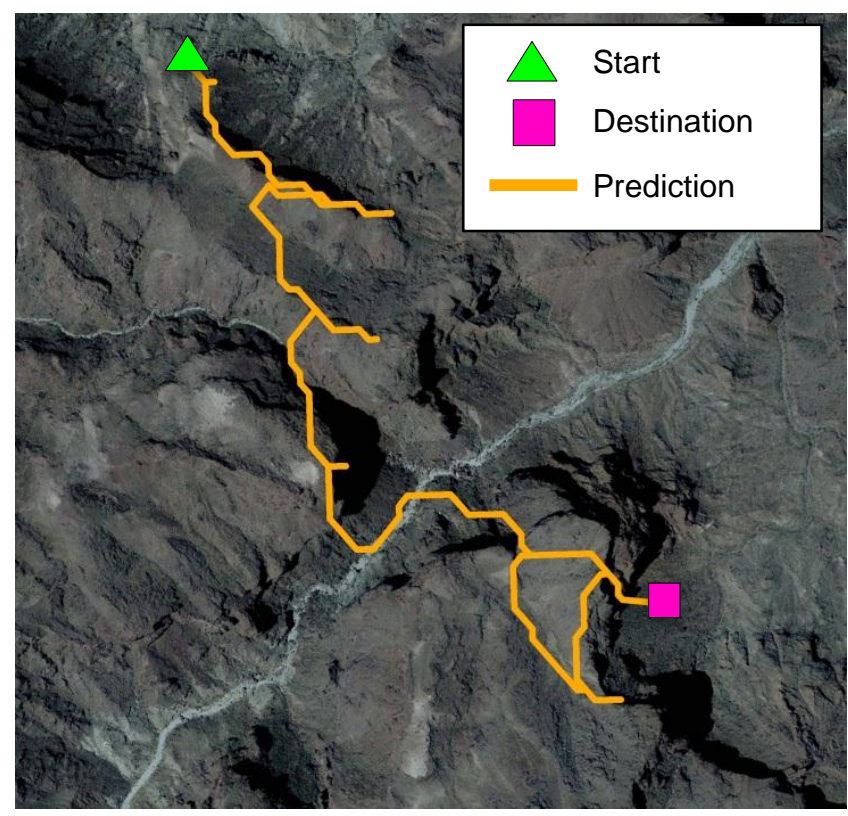

Figure 6-1: The control simulation.

\subsection{Terrain Influences}

Changes in elevation and terrain affect the viewshed calculation. Since viewshed affects the determined path, changes in elevation should alter simulation output. To determine these effects, a DEM representing elevation in the southwestern US was used; this surface will be referred to as "original" or control. To keep other variables constant, the original DEM was smoothed using a low-pass filter; and this surface will be referred to as "medium." When a low-pass filter is applied to the DEM, elevation values are averaged based on a 3x3 kernel. To get an even smoother surface, the low-pass filter was applied to the medium surface; this surface will be referred to as "flat." The calculation results from the three terrains are listed in Table 3. 
Table 3. Analysis effects from surface variation.

\begin{tabular}{|c|c|c|c|c|}
\hline $\begin{array}{l}\text { Recalculation } \\
\text { Percentage }\end{array}$ & $\begin{array}{l}\text { Height } \\
\text { (m) }\end{array}$ & $\begin{array}{l}\text { Number of } \\
\text { Eval. Points }\end{array}$ & Elevation Surface & Total Time(min) \\
\hline 80 & 2 & 14 & original & 8.33 \\
\hline 80 & 2 & 21 & medium & 14.4 \\
\hline 80 & 2 & 25 & flat & 27.5 \\
\hline
\end{tabular}

Comparing the control simulation and the simulation using the medium surface, the two resulting paths have the same general trend, but at some location the control simulation turned around while the medium surface path continued. The path from the medium surface is presented in Figure 6-2 as the purple line and the control simulation is represented with the orange line.

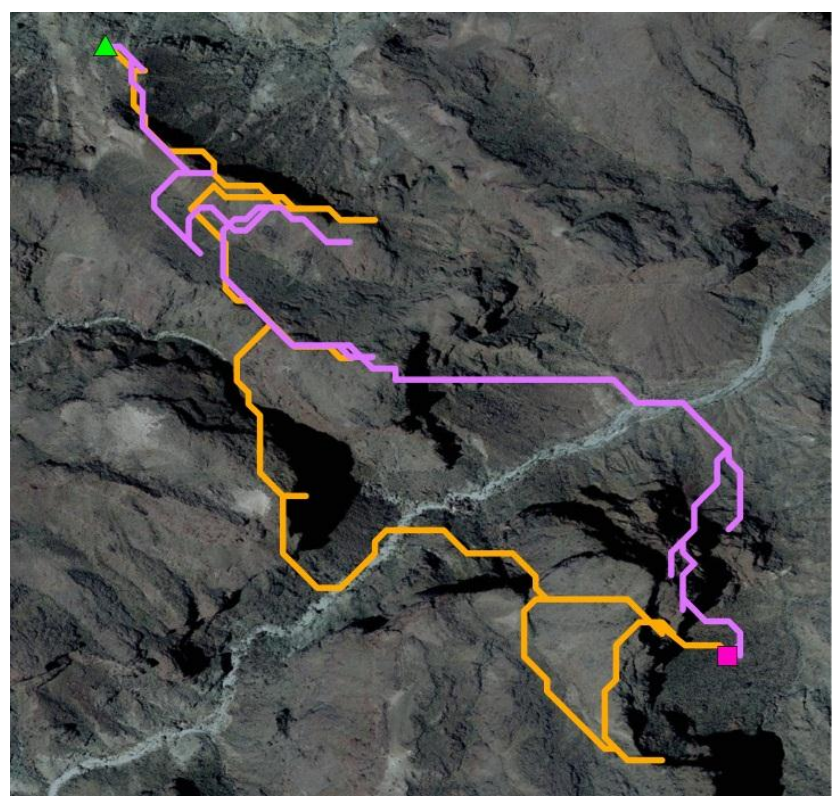

\section{Figure 6-2: Medium surface results.}

Referring to Table 3, more differences are apparent. The medium surface required 21 evaluation points and over 14 minutes of processing time. Since analysis was restricted to viewshed, and visibility was increased with the medium surface, one would suspect a faster simulation and fewer recalculation points from the medium surface, but the opposite was true.

Figure 6-3 shows the regular predicted path in orange and the flat surface prediction in purple. The differences in the path are visible again; the flat surface simulation decided upon a more direct route. 


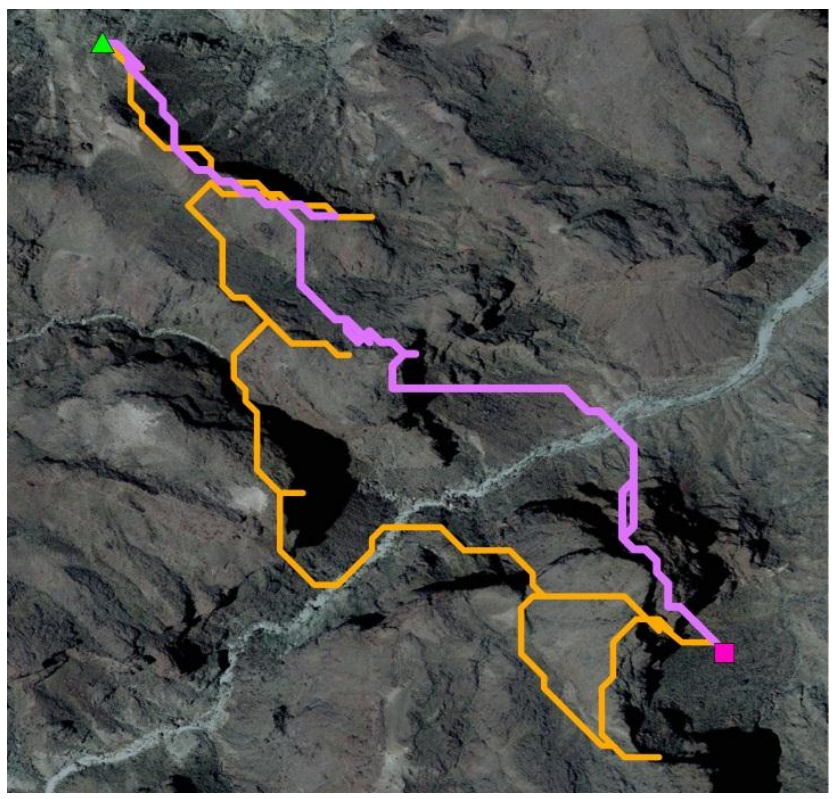

Figure 6-3: Flat surface results.

Referring to Table 3, the flat surface required 25 recalculation points, which was unexpected because the smoother surface should allow for a larger visible area thus a longer distance travel per iteration but again the opposite was true.

This comparison proves that a prediction cannot be made about the number of evaluation points or evaluation time on terrain because each scenario was different.

\subsection{Recalculation Percentages}

Recalculation percentage can be interpreted as speed, in that if a person is moving slower they might make more decisions or reconsider their route sooner. This can be equated to driving a car; when a person is driving $25 \mathrm{mph}$ it is easier to make a turn or find a street as opposed to a driver traveling at $65 \mathrm{mph}$. To evaluate recalculation differences, one simulation was run with a $60 \%$ recalculation and another with $100 \%$ recalculation. All three simulations will use the original DEM. Recalculation percentage is the percentage of the determined path that will be traveled before stopping to make another evaluation.

Figure 6-4 shows the differences between a 60 percentage and an 80 percentage recalculations, the purple and orange lines respectively. The purple path diverges significantly from the orange control path. 


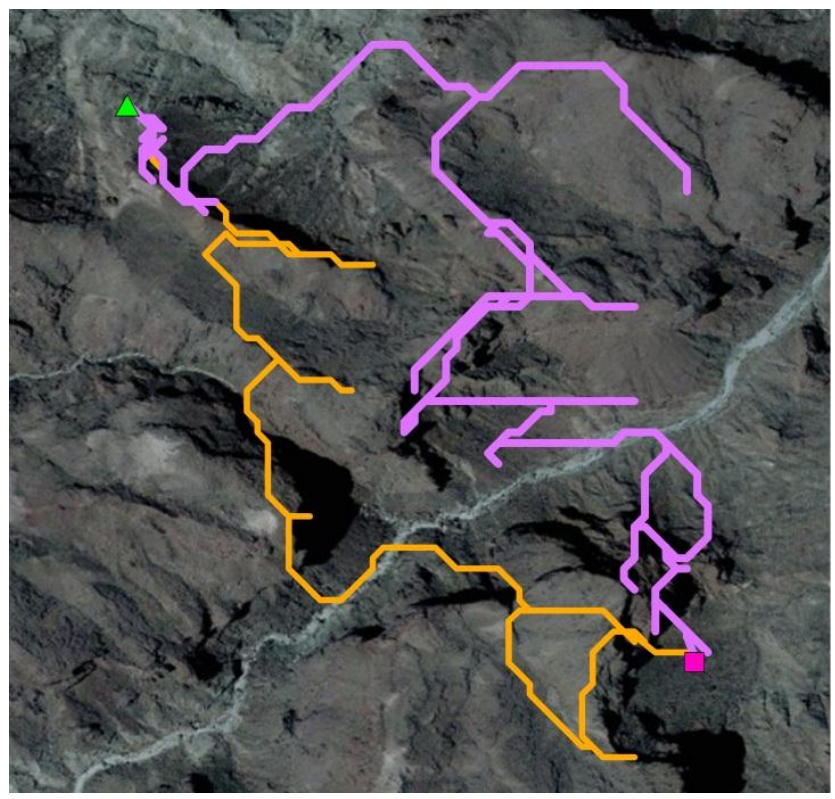

Figure 6-4: Difference between a $60 \%$ and an $80 \%$ recalculation.

Comparing the numbers from Table 4 , the $60 \%$ recalculation simulation had 28 evaluation points whereas the control had 14 . Processing time for the $60 \%$ simulation was longer at over 30 minutes against the control's, a little over eight minutes.

Table 4. Difference in recalculation percentage

\begin{tabular}{|r|r|l|l|r|}
\hline $\begin{array}{l}\text { Recalculation } \\
\text { Percentage }\end{array}$ & $\begin{array}{l}\text { Height } \\
(\mathrm{m})\end{array}$ & $\begin{array}{l}\text { Number of Eval. } \\
\text { Points }\end{array}$ & $\begin{array}{l}\text { Elevation } \\
\text { Surface }\end{array}$ & Total Time(min) \\
\hline 100 & 2 & 8 & regular & 13.6 \\
\hline 80 & 2 & 14 & regular & 8.33 \\
\hline 60 & 2 & 28 & regular & 30.7 \\
\hline
\end{tabular}

The $100 \%$ recalculation path only had 8 evaluation points to the control's 14 , but a longer processing time, over 13 minutes. The longer analysis time is interesting because the code used for the $100 \%$ recalculation should be more efficient, additionally the $100 \%$ path required fewer evaluations. Figure 6-5 presents the $100 \%$ path in purple and the control path in orange. The two paths were similar for short distance, but where the orange route turned around, the purple path continued. 


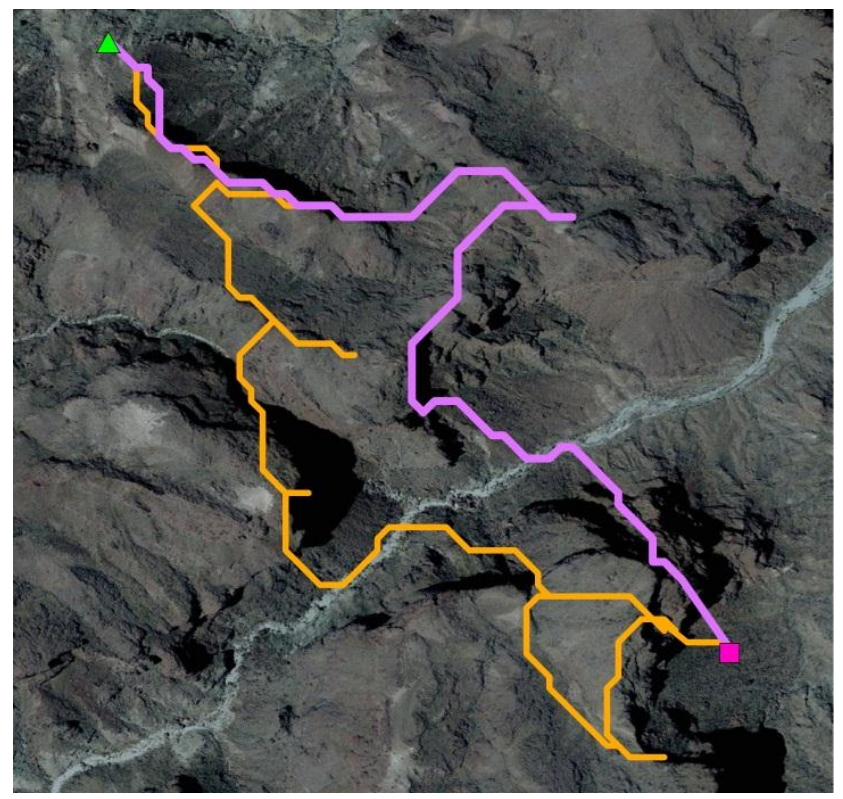

Figure 6-5: Comparing an $80 \%$ and a $100 \%$ recalculation.

\subsection{Directional Bias}

The directional bias functionality provides the simulation with the knowledge of a preferred direction. This can dramatically alter the predicted path. For this analysis, the travel paths from Section 6.2 were performed again with the additional of a $60^{\circ}$ directional bias and an additional cost of one applied to the non-preferred direction.

Figure 6-6 shows the effects of the directional bias on the $60 \%$ recalculation path, the original path is purple and the directional bias path is orange. Notice how the purple path diverges while the orange path follows a more direct route. 


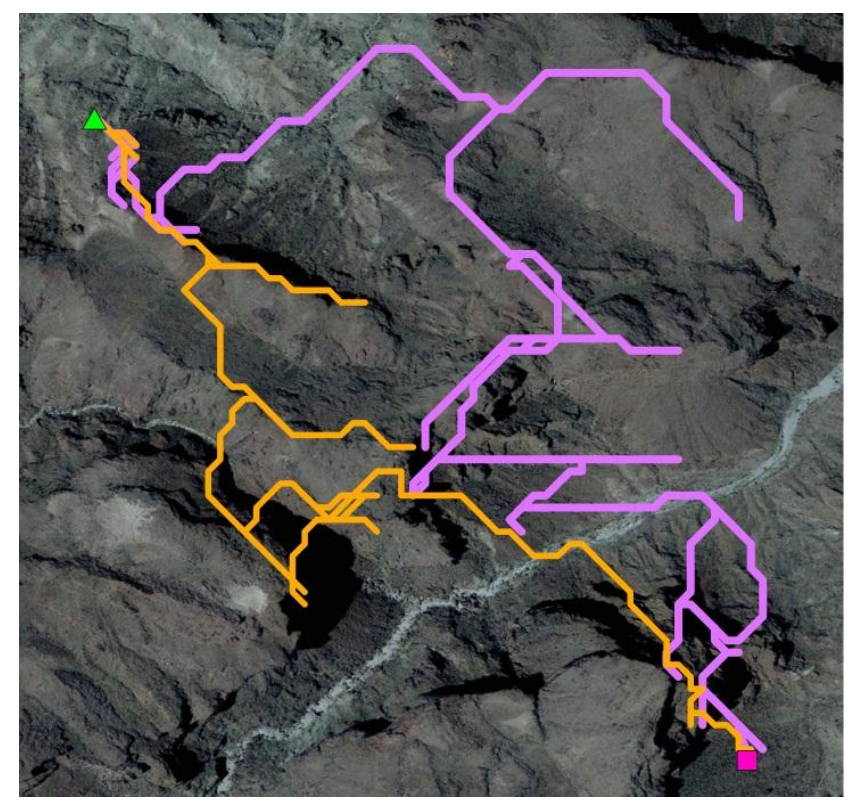

Figure 6-6: Comaparing 60\% recalculation with a directional bias.

Table 5 reveals more of the differences; the orange path required more evaluation points but had a shorter processing time.

Table 5. Comparing paths with and without directional bias.

\begin{tabular}{|r|r|l|l|r|r|}
\hline $\begin{array}{l}\text { Recalculation } \\
\text { Percentage }\end{array}$ & $\begin{array}{l}\text { Height } \\
(\mathrm{m})\end{array}$ & $\begin{array}{l}\text { Number of Eval } \\
\text { Points }\end{array}$ & $\begin{array}{l}\text { Elevation } \\
\text { Surface }\end{array}$ & $\begin{array}{l}\text { Total } \\
\text { Time(min) }\end{array}$ & $\begin{array}{l}\text { Directional } \\
\text { Bias/Value }\end{array}$ \\
\hline 60 & 2 & 41 & Regular & 24.7 & $60 / 1$ \\
\hline 60 & 2 & 28 & Regular & 30.7 & 0 \\
\hline 80 & 2 & 26 & Regular & 27.8 & $60 / 1$ \\
\hline 80 & 2 & 14 & Regular & 8.33 & 0 \\
\hline 100 & 2 & 8 & Regular & 8.67 & $60 / 1$ \\
\hline 100 & 2 & 8 & Regular & 13.6 & 0 \\
\hline
\end{tabular}

Figure 6-7 presents the differences in the $80 \%$ recalculation with a directional bias in orange and without the bias in purple. Upon visual inspection, the paths are noticeably different but one does not appear to be more direct or efficient than the other. Referring back to Table 5, the purple path required almost double the evaluation points and required significantly more processing time. 


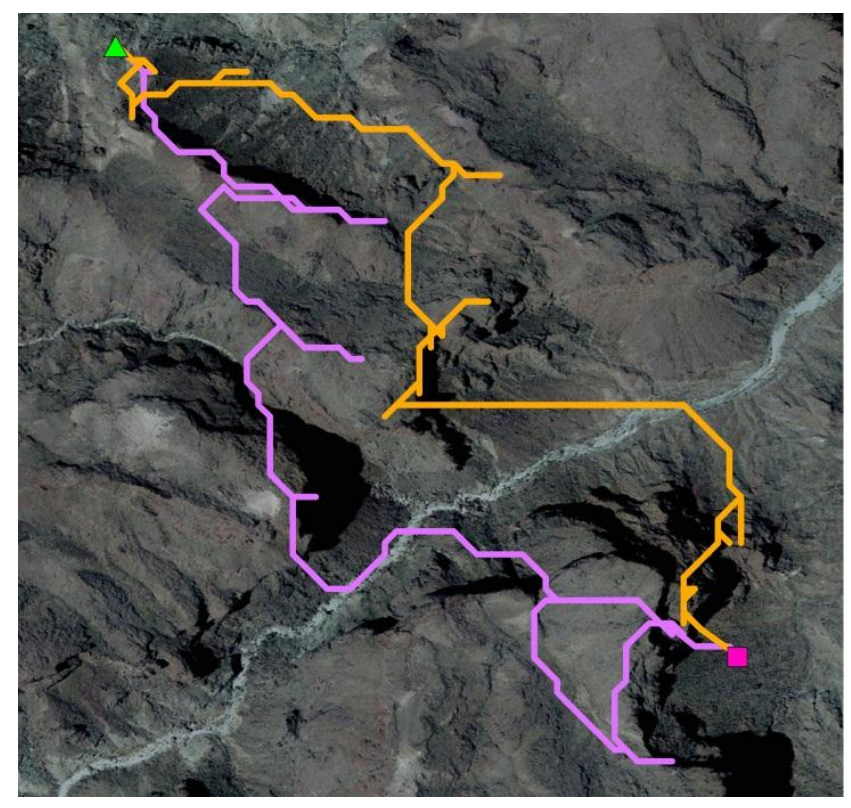

Figure 6-7: Comaparing $80 \%$ recalculation with a directional bias.

Figure 6-8 presents the comparison on the $100 \%$ recalculation path with directional bias in orange and without the bias in purple. The two paths follow almost exactly the same route. Referring to Table 5 reveals that the orange and the purple paths had the same number of recalculation points but the purple path had a shorter processing time.

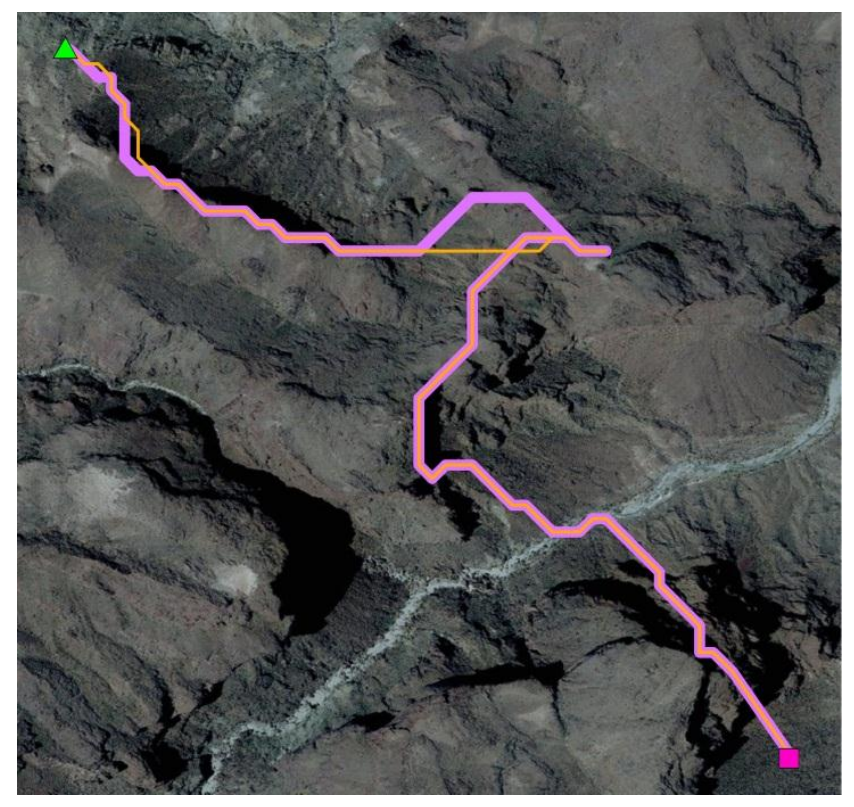

Figure 6-8: Comaparing $100 \%$ recalculation with a directional bias. 
The results from this section provide further evidence that the simulation is very difficult to predict. There appears to be a trend in the paths from significantly different in the $60 \%$ comparison to almost identical in the $100 \%$ paths.

\subsection{Standard Least Cost Path}

As mentioned previously, a standard LCPA has access to the cost surface of the entire study area. With that, the path prediction tool utilizes a LCPA to predict where a person will travel, so one would expect some similarities. However, a visual comparison of the orange control simulation to the blue standard LCPA (Figure 6-9) revealed the two determined routes are quite different.

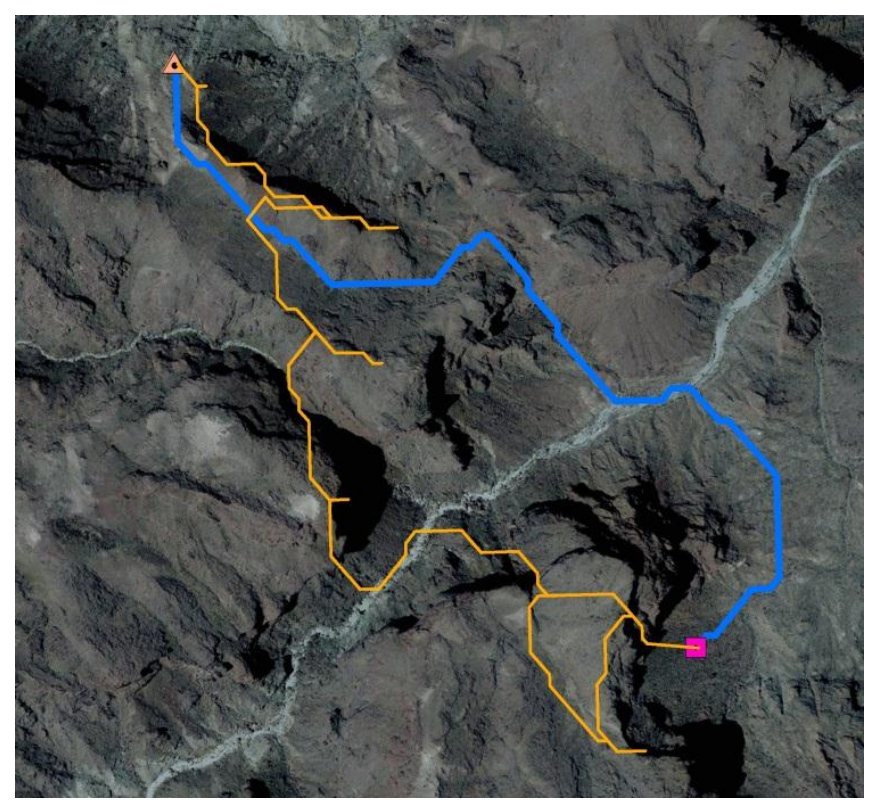

Figure 6-9: Comparing an $80 \%$ recalculation to a standard LCPA.

Further differences are observed when comparing processing time (Table 6). The simulation took over 14 minutes to process whereas the standard LCPA took approximately 16 seconds. Additional field testing is required to verify if the path prediction tool is more effective in this task than the unrestricted LCPA.

Table 6. Comparing a standard LCPA with the control simulation

\begin{tabular}{|r|r|l|l|r|}
\hline $\begin{array}{l}\text { Recalculation } \\
\text { Percentage }\end{array}$ & Height (m) & $\begin{array}{l}\text { Number of } \\
\text { Eval. Points }\end{array}$ & $\begin{array}{l}\text { Elevation } \\
\text { Surface }\end{array}$ & Total Time(min) \\
\hline N/A & N/A & 1 & regular & 0.267 \\
\hline 80 & 2 & 14 & regular & 8.33 \\
\hline
\end{tabular}




\subsection{Observer Heights}

Observer height is a key component in viewshed calculation because it affects the field of view. The path prediction tool increases observer height when little or no progress is made to create a larger viewshed so the simulation can progress. For analysis purposes, observer heights of $1.5 \mathrm{~m}$ and $2.5 \mathrm{~m}$ were compared to the control $2 \mathrm{~m}$. Both the $1.5 \mathrm{~m}$ and $2.5 \mathrm{~m}$ observer heights produced paths identical to the control. However, there were differences in analysis time (Table 7); the control simulation had the fastest processing time. The half meter changes in observer height were not enough to alter the VSA output. The exact threshold for viewshed change is uncertain.

Table 7. Comparing simulations with different observer heights.

\begin{tabular}{|r|r|l|l|r|}
\hline $\begin{array}{l}\text { Recalculation } \\
\text { Percentage }\end{array}$ & $\begin{array}{l}\text { Height } \\
(\mathrm{m})\end{array}$ & $\begin{array}{l}\text { Number of Eval. } \\
\text { Points }\end{array}$ & $\begin{array}{l}\text { Elevation } \\
\text { Surface }\end{array}$ & Total Time(min) \\
\hline 80 & 2.5 & 14 & regular & 8.85 \\
\hline 80 & 2 & 14 & regular & 8.33 \\
\hline 80 & 1.5 & 14 & regular & 11.37 \\
\hline
\end{tabular}

\subsection{Problems with Viewshed Analysis}

The analysis results showed there was a problem with the simulation, resulting in gaps in the determined path. The problem was traced to an issue with ArcMap's VSA tool. The tool encounters problems when converting from vector data to raster. Figure 6-10 is an instance of ArcMap failing to accurately convert from vector to raster. A VSA is performed on the point (purple triangle), but the cell it occupies was determined to be non-visible.

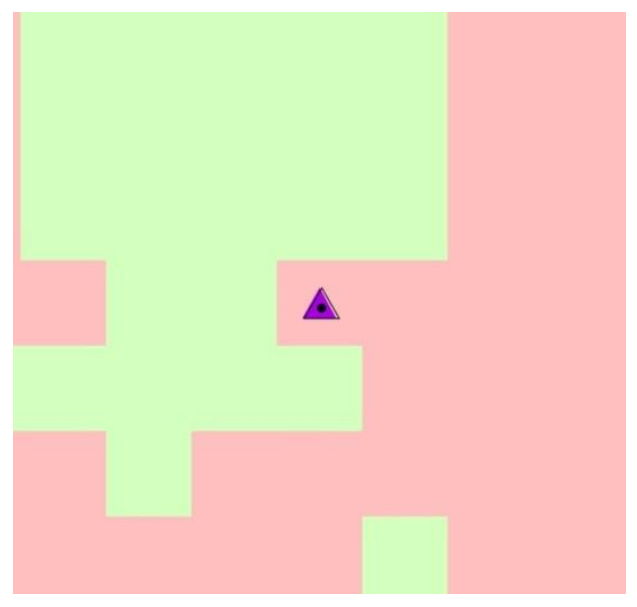

Figure 6-10: A failed viewshed analysis. 
This error can result in a failed analysis because all data in the non-visible area is clipped, including the determined path. In Figure 6-10, for example, if part of the determined path traveled through the pink, non-visible area then it will be discarded by the program and create gaps in the determined path or cause the program to crash. A subroutine was implemented to verify that the occupied cell was visible. To do this, the viewshed result is converted to a matrix of numbers. Then the evaluation point coordinates are converted to row and column numbers corresponding with the matrix. The program locates the evaluation point cell in the matrix and ensures the cell was visible, if it was not then the program will alter the cell value appropriately.

\subsection{Summary}

As the analysis proves, there are many factors that influence the tool's output and few factors that can be used to predict analysis performance. Adjusting the recalculation percentage is akin to a traveler's level of confidence or speed. Next, the addition of a directional bias created a more intelligent simulation in that it knows where it should travel, and takes a more direct route. Finally, the results from the height analysis proved that small changes in height produce little to no change in the program prediction but did augment the analysis time.

It remains to be seen if the program can accurately predict human travel through an environment. However, the results resemble more human characteristics of travel conveyed in the travel predictions where the traveler turned around. By performing these analyses, the program performed simulations on various situations and revealed weaknesses in the program that were addressed. For example, an issue with VSA was discovered and a workaround was implemented. The analyses promoted the importance of the cost surface and how the cost surface controls the simulation behavior. This characteristic was most prominent in the directional bias analysis, wherein the cost surface was modified to prefer a direction towards the destination. The addition of direction to the cost surface significantly changed the $60 \%$ recalculation path - arguably for the better. For future users of the tool, the cost surface should be a major concern. 


\section{Chapter 7 - Conclusions and Future Work}

Chapter 7 discusses conclusions and future work for the path prediction tool. Section 7.1 provides a summary of the project and client feedback, and Section 7.2 discusses future work.

\subsection{Conclusion}

The goal of this project was to create a path prediction tool that included the following components: least cost path restricted by viewshed, directional bias, variable evaluation percentage, and a cost surface randomization capability. All of these components were included. The final product performed well because the directional bias functionality works dynamically with each evaluation point and the model outputs the considered paths. Considered paths were not an original requirement; however, when consulting with the client he agreed they could be valuable.

While the analysis performed in Chapter 6 provided some insight to the simulation, the ultimate conclusion was that it is difficult to predict how the program will navigate through an environment, and because of this it is also difficult to forecast analysis time. A change in a single decision point by as little as a single cell will produce a different prediction altogether. And without access to data of a real scenario for comparison, it is difficult to say how well the simulation will perform.

The program will certainly not be able to predict the exact route a person will travel, but the prediction may provide insight to a traveler's overall trend. With that, questions arise on the importance of high resolution data. It may be more beneficial in terms of analysis time and resources to use lower resolution data to produce an overall trend rather than a step-by-step prediction. The user could input lower resolution data and derive a prediction faster, and then allocate resources to a broader area while still achieving a level of efficiency. It's a matter of weighing the costs and benefits of the operation.

Predicting human decision-making and travel is a difficult task because there are numerous externalities that need to be accounted for. While this simulation provides a tool to predict human behavior, it is ultimately the responsibility of a user to interpret the final results.

\subsection{Future Work}

While the simulation accounts for numerous inputs, more variables can be added. One idea is to have decision-making break down over time to simulate human fatigue and frustration. The current model simulated decision-making over a constant travel percentage. Incorporating this into the model would require research in human psychology to find an equation to describe the breakdown of cognition over time. This addition would accompany the model with a database containing variable presets so the user would need only to select an option from a drop-down menu. For example, given a search and rescue situation, the approach for locating a healthy 18 -year-old male will be different than the approach for a 70-year-old Alzheimer's patient. The 18-year-old will 
have the capacity to move further in the same amount of time and make logical decisions, compared to the Alzheimer's patient who will travel slower and make more unpredictable decisions.

The client requested the capability to model visibility restrictions from vegetation and structures. To include this aspect would require the creation of a new viewshed tool that produces a probabilistic viewshed as described by Riggs and Dean (2007). Instead of a binary output, the probabilistic viewshed would assign probabilities to a cell. If a probabilistic viewshed tool was created, the path prediction tool would require a new cost surface restriction approach to account for a fuzzy viewshed. To determine the permeability of light through different types of vegetation would require a comprehensive analysis of vegetation density, transparency, and seasonal changes.

The program is still considered to be in the prototype stage primarily because of the lengthy processing time. The two components that require the most time to perform are the LCPA (about 45 seconds per iteration) and VSA (about 20 seconds per iteration). That is a combined total of 65 seconds per iteration for an area of 5 square miles. When an analysis requires over 100 iterations, processing time accumulates to almost two hours. There are ways to decrease run time, such as decreasing the resolution of the DEM and cost surface so the computer does not have as much data to process, and/or increase the observer height leading to fewer iterations. However, both of these improvements will degrade the final result and increasing the visible area does not always equate to faster processing times, as proven in Chapter 6. ESRI, maker of ArcMap, released a new version of their mapping software, ArcMap 10.1, and claims it has the ability to perform LCPA and VSA up to 30 times faster. The simulation was not constructed on this platform because development began prior to the ArcMap 10.1 release.

Up to this point, the simulation has been tested on a laptop computer, but if the final end-user is operating on a more powerful laptop or desktop computer, they will conceivably see faster processing times. Another way to improve performance is to compile the code or write the program in a different language, such as $\mathrm{C}++$ because compiled code runs much faster than interpreted Python code.

For the analysis in Chapter 6, a cost surface was used based on slope. However, an understanding of the study area and the traveler is required for constructing an accurate cost surface to produce a highly probable result. It is the responsibility of a domain expert to generate such cost surfaces because of the range of responses a real person will have to the environment. For example, water bodies can be classified with high or low cost depending on temperature, food and water availability, and the individual's capacity to swim. Furthermore, constructing a cost surface for this simulation is different from constructing a cost surface for an unrestricted least cost path because of the perspective change in the simulation. The surface used in the Chapter 6 analysis used slope, and a higher cost was applied to steeper slopes. However, that value remains the same when traveling up or down an incline when in reality perception of difficulty will change and a person may believe it is easier to travel down-hill. 


\section{Works Cited}

Bagli, S., Geneletti, D., \& Orsi, F. (2011, April). Routeing of power lines through leastcost path analysis and multicriteria evaluation to minimise environmental impacts. Environmental Impace Assessment Review, pp. 234-239.

Carlisle, B. H. (2005). Modeling the spatial distribution of DEM error. Transactions in GIS, 521-540.

Collischonn, W., \& Pilar, J. V. (2000, June). A direction dependent least cost path algorith for roads and canals. Retrieved February 20, 2012, from Universidad Nacional Del Nordeste Facultad De Ingerieria: http://arandu.org.ar/pub/pd-gis.pdf

Delavar, M., \& Naghibi, F. (2003). Pipeline routing using geospatial information system analysis. The Scan GIS Conference Series. Espoo, Finland: Helsinki University of Technology.

Jobe, R. T., \& White, P. S. (2009). A new cost-distance model for human accessibility and an evaluation of accessibility bias in permanent vegetation plots in Great Smokey Mountains National Park, USA. Journal of Vegetation Science, 10991109.

Kim, Y.-H., Rana, S., \& Wise, S. (2004). Exploring multiple viewshed analysis using terrain features and optimisation techniques. Computers and Geosciences, 10191032.

Kumler, M. P. (1994). An Intensive Comparison of Triangulated Irregular Networks (TINs) and Digital Elevation Models (DEMs). Cartographica: The international Journal for Geographic Information Visualization, 1-99.

Le Yaouanc, J.-M., Saux, E., \& Claramunt, C. (2010). A visibility and spatial constraintbased approach for geopositioning. Geographic Information Science, Lecture Notes in Computer Science, Volume 6292.

Lee, J., \& Stucky, D. (1998). On applying viewshed analysis for determing least-cost paths on Digital Elevation Models. International Journal of Geographical Information Science, 891-905.

Riggs, P. D., \& Dean, D. J. (2007). An investigation into the causes of errors and inconsistencies in predicted viewsheds. Transactions in GIS, Vol 11 175-196.

Rothley, K. (2005). Finding and filling the "cracks" in resistance surfaces for least-cost modeling. Ecology and Society, Vol 10.

Saha, A. K., Arora, M. K., Gupta, R. P., Virdi, M. L., \& Csaplovics, E. (2005). GISbased route planning in landslide-prone areas. International Journal of Geographic Infromation Science, Vol 19 No. 10 1149-1175.

Walker, R., \& Craighead, L. (1997). Analyzing Wildlife Movement Corridors in Montana. Retrieved February 2012, from ESRI: proceedings.esri.com

Wilson, J. P., \& Gallant, J. C. (2000). Terrain Analysis: Principles and Applications. John Wiley and Sons, Inc. 



\section{Appendix A.}

This section contains information about specific code sequences that were not included in other chapters.

\section{A.1 Addressing Viewshed Error}

To cope with the VSA error, the code verifies directly if the viewshed output identified the occupied cell as visible. Figure A-1 contains the code snippet that performs this action.

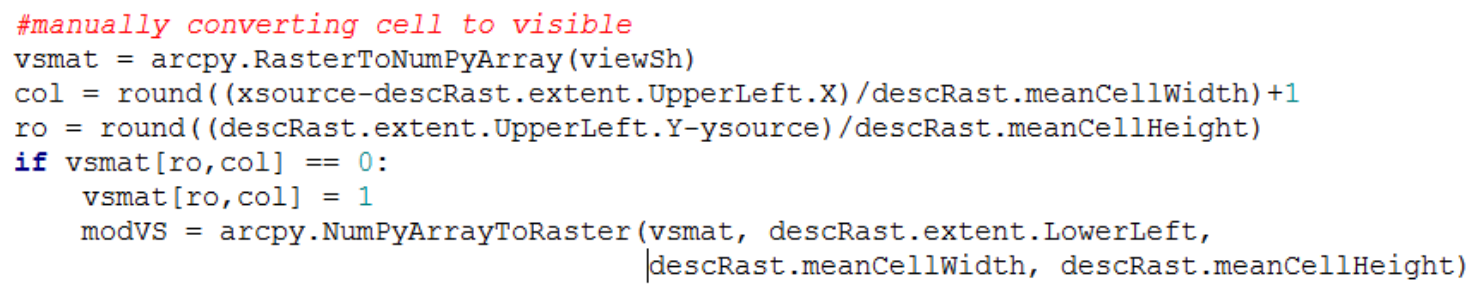

Figure A-1: Code used to verify VSA results.

\section{A.2 Handling Discontinuity}

Occasionally the raster to vector conversion would create single-celled discontinuities in the resulting path (Figure A-2). In the figure, A presents a path with single-celled gaps and $\mathrm{B}$ presents a continuous path. To make the final path aesthetically more pleasing, the program performs a test (Figure A-3) to identify if there is a gap and connects the points. If the beginning of the next line is over half a cell away from the current evaluation point, then the program will connect the two.

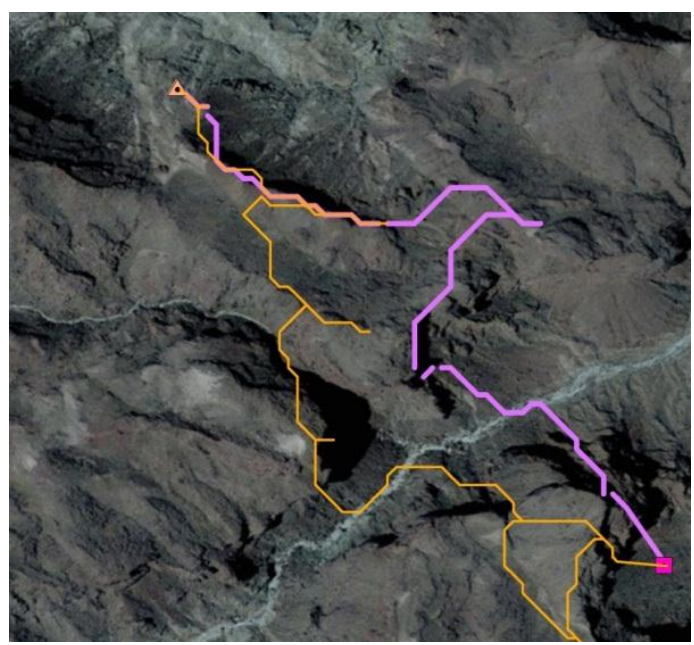

(A)

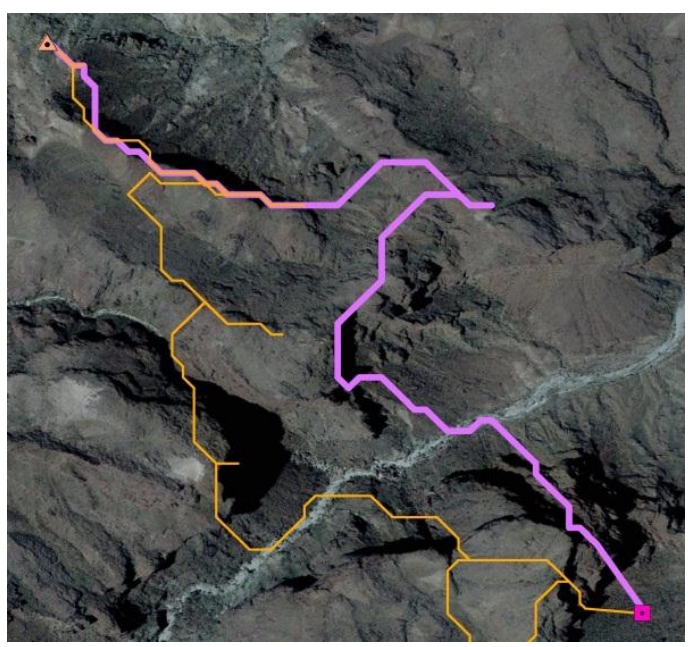

(B)

Figure A-2: Single cell gaps. 


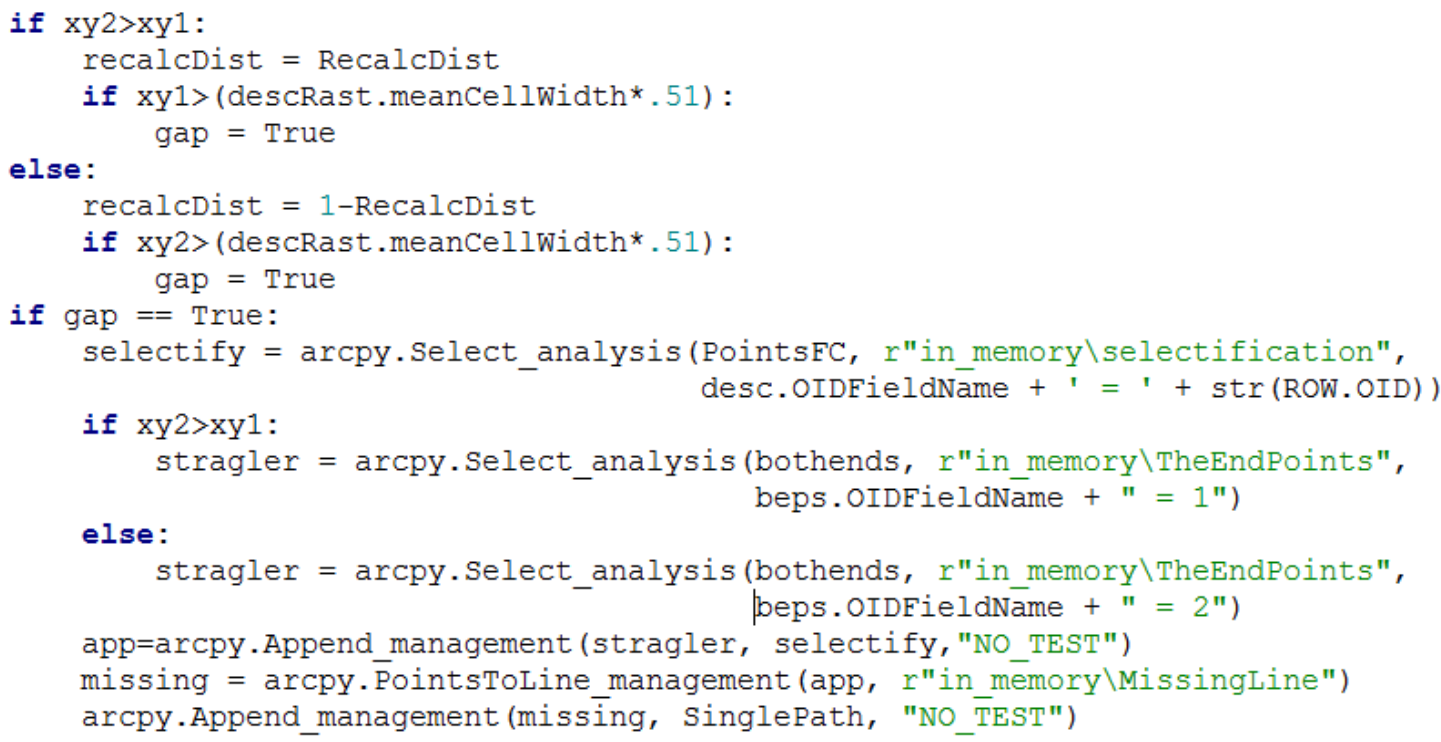

Figure A-3: Code used to patch gaps in the determined path. 